\title{
YETTER-DRINFELD CATEGORIES FOR QUASI-HOPF ALGEBRAS
}

\author{
D. BULACU, S. CAENEPEEL, AND F. PANAITE
}

\begin{abstract}
We show that all possible categories of Yetter-Drinfeld modules over a quasi-Hopf algebra $H$ are isomorphic. We prove also that the category ${ }_{H}^{H} \mathcal{Y} D^{\mathrm{fd}}$ of finite dimensional left Yetter-Drinfeld modules is rigid and then we compute explicitly the canonical isomorphisms in ${ }_{H}^{H} \mathcal{Y} D^{\mathrm{fd}}$. Finally, we show that certain duals of $H_{0}$, the braided Hopf algebra introduced in [6] 7], are isomorphic as braided Hopf algebras if $H$ is a finite dimensional triangular quasi-Hopf algebra.
\end{abstract}

\section{INTRODUCTION}

Let $H$ be a Hopf algebra with a bijective antipode. We can introduce left, right, left-right and right-left Yetter-Drinfeld modules over $H$, and it is well-known (see [2] 17]) that the corresponding categories ${ }_{H}^{H} \mathcal{Y} D, \mathcal{Y} D_{H}^{H},{ }_{H} \mathcal{Y} D^{H}$ and ${ }^{H} \mathcal{Y} D_{H}$ are isomorphic. These categories are also isomorphic to the center of the monoidal category ${ }_{H} \mathcal{M}$ of left $H$-modules, and, if $H$ is finite dimensional, to the category $D(H) \mathcal{M}$ of left modules over the Drinfeld double $D(H)$. It is also known that the category of finite dimensional Yetter-Drinfeld modules is rigid, that is, we have left and right duality in this category.

In [10, Dijkgraaf, Pasquier and Roche introduced the so-called "twisted double" of a finite group, which is a Hopf algebra-type object $D^{\omega}(G)$ associated to a pair $(G, \omega)$, where $G$ is a finite group and $\omega$ is a normalized 3-cocycle on $G$; this object is not a Hopf algebra, but a quasi-Hopf algebra in the sense of Drinfeld [11. The construction is similar to the quantum double, so it appears natural to try to define the quantum double of an arbitrary finite dimensional quasi-Hopf algebra, generalizing the Drinfeld double for Hopf algebras, and then to show that $D^{\omega}(G)$ is such a quantum double. This has been done first by Majid in [15]; he first computed the center of the monoidal category ${ }_{H} \mathcal{M}$ of left $H$-modules over the quasi-Hopf algebra $H$ (we will denote this center by ${ }_{H}^{H} \mathcal{Y} D$ and call its objects left Yetter-Drinfeld modules over $H$ ). Then he defined the quantum double $D(H)$ by an implicit Tannaka-Krein reconstruction procedure, in such a way that ${ }_{D(H)} \mathcal{M} \cong$ ${ }_{H}^{H} \mathcal{Y} D$. An explicit construction of the quantum double (as a so-called "diagonal crossed product") has been given afterwards by Hausser and Nill in [12, 13. They

1991 Mathematics Subject Classification. 16W30.

Key words and phrases. quasi-Hopf algebra, Yetter-Drinfeld module, braided monoidal category.

Research supported by the bilateral project "Hopf Algebras in Algebra, Topology, Geometry and Physics" of the Flemish and Romanian governments. This paper was finished while the first author was visiting the Vrije Universiteit Brussel, and he would like to thank VUB for its warm hospitality. The third author was also partially supported by the programmes SCOPES and EURROMMAT. 
identified the category of left modules over their quantum double with the category ${ }_{H^{\mathrm{cop}}}^{\mathrm{cop}} \mathcal{Y} D$ (we will denote this category by ${ }_{H} \mathcal{Y} D^{H}$ and call its objects left-right YetterDrinfeld modules over $H$ ). Now the question arises whether ${ }_{H}^{H} \mathcal{Y} D \cong{ }_{H} \mathcal{Y} D^{H}$.

The first aim of this paper is to show that, indeed, the categories ${ }_{H}^{H} \mathcal{Y} D$ and ${ }_{H} \mathcal{Y} D^{H}$ (and also two other categories $\mathcal{Y} D_{H}^{H}$ and ${ }^{H} \mathcal{Y} D_{H}$ which we will introduce) are isomorphic, even in the situation where $H$ is not finite dimensional. In an earlier version of this paper, a computational proof of this result was given, which was much more complicated than the corresponding proof for Yetter-Drinfeld modules over coassociative Hopf algebras. Viewing the categories of Yetter-Drinfeld modules as (left or right) centers of corresponding categories of modules, a more transparent approach is possible, and this is what we will do in Section 2 This approach was suggested to us by the referee.

In Section 3 we show that the category ${ }_{H}^{H} \mathcal{Y} D^{\mathrm{fd}}$ of finite dimensional (left) YetterDrinfeld modules is rigid; the left and right duals are constructed explicitly. In an arbitrary rigid braided monoidal category $\mathcal{C}$, we have canonical isomorphisms $M \cong M^{* *}$ and $(M \otimes N)^{*} \cong M^{*} \otimes N^{*}$. In Section 4 we compute these isomorphisms in the case $\mathcal{C}={ }_{H}^{H} \mathcal{Y} D^{\mathrm{fd}}$. If we then specialize to finite dimensional left modules over a quasitriangular quasi-Hopf algebra, we recover some results from [8].

Let $B$ be a braided Hopf algebra, that is a Hopf algebra in ${ }_{H}^{H} \mathcal{Y} D^{\mathrm{fd}}$. Then $B^{*}$ and ${ }^{*} B$ are also braided Hopf algebras. In Section [5 we study the special case where $B=H_{0}$, the braided Hopf algebra introduced in 6] 7]. In particular, we prove that ${ }^{*} H_{0}$ and $H_{0}^{*}$ are isomorphic braided Hopf algebras if $H$ is a finite dimensional triangular quasi-Hopf algebra. They are also isomorphic to the coopposite of the braided Hopf algebra $\underline{H}^{*}$ introduced in $\underline{3}$.

\section{Preliminary Results}

1.1. Quasi-Hopf algebras. We work over a commutative field $k$. All algebras, linear spaces etc. will be over $k$; unadorned $\otimes$ means $\otimes_{k}$. Following Drinfeld [11, a quasi-bialgebra is a fourtuple $(H, \Delta, \varepsilon, \Phi)$ where $H$ is an associative algebra with unit, $\Phi$ is an invertible element in $H \otimes H \otimes H$, and $\Delta: H \rightarrow H \otimes H$ and $\varepsilon: H \rightarrow k$ are algebra homomorphisms satisfying the identities

$$
\begin{aligned}
& (i d \otimes \Delta)(\Delta(h))=\Phi(\Delta \otimes i d)(\Delta(h)) \Phi^{-1}, \\
& (i d \otimes \varepsilon)(\Delta(h))=h, \quad(\varepsilon \otimes i d)(\Delta(h))=h,
\end{aligned}
$$

for all $h \in H$. $\Phi$ has to be a normalized 3-cocycle, in the sense that

$$
\begin{aligned}
& (1 \otimes \Phi)(i d \otimes \Delta \otimes i d)(\Phi)(\Phi \otimes 1)=(i d \otimes i d \otimes \Delta)(\Phi)(\Delta \otimes i d \otimes i d)(\Phi), \\
& (i d \otimes \varepsilon \otimes i d)(\Phi)=1 \otimes 1 .
\end{aligned}
$$

The map $\Delta$ is called the coproduct or the comultiplication, $\varepsilon$ the counit and $\Phi$ the reassociator. As for Hopf algebras [19] we denote $\Delta(h)=h_{1} \otimes h_{2}$ (summation understood), but since $\Delta$ is only quasi-coassociative we adopt the further convention

$$
(\Delta \otimes i d)(\Delta(h))=h_{(1,1)} \otimes h_{(1,2)} \otimes h_{2}, \quad(i d \otimes \Delta)(\Delta(h))=h_{1} \otimes h_{(2,1)} \otimes h_{(2,2)},
$$

for all $h \in H$. We will denote the tensor components of $\Phi$ by capital letters, and the ones of $\Phi^{-1}$ by small letters, namely

$$
\begin{aligned}
& \Phi=X^{1} \otimes X^{2} \otimes X^{3}=T^{1} \otimes T^{2} \otimes T^{3}=V^{1} \otimes V^{2} \otimes V^{3}=\cdots \\
& \Phi^{-1}=x^{1} \otimes x^{2} \otimes x^{3}=t^{1} \otimes t^{2} \otimes t^{3}=v^{1} \otimes v^{2} \otimes v^{3}=\cdots
\end{aligned}
$$


$H$ is called a quasi-Hopf algebra if, moreover, there exists an anti-automorphism $S$ of the algebra $H$ and elements $\alpha, \beta \in H$ such that, for all $h \in H$ :

$$
\begin{aligned}
& S\left(h_{1}\right) \alpha h_{2}=\varepsilon(h) \alpha \quad \text { and } \quad h_{1} \beta S\left(h_{2}\right)=\varepsilon(h) \beta, \\
& X^{1} \beta S\left(X^{2}\right) \alpha X^{3}=1 \quad \text { and } \quad S\left(x^{1}\right) \alpha x^{2} \beta S\left(x^{3}\right)=1 .
\end{aligned}
$$

Note that, in Drinfeld's original definition, the antipode of a quasi-Hopf algebra is required to be bijective. The axioms for a quasi-Hopf algebra imply that $\varepsilon(\alpha) \varepsilon(\beta)=$ 1 , so, by rescaling $\alpha$ and $\beta$, we may assume without loss of generality that $\varepsilon(\alpha)=$ $\varepsilon(\beta)=1$ and $\varepsilon \circ S=\varepsilon$. The identities (1.21.4) also imply that

$$
(\varepsilon \otimes i d \otimes i d)(\Phi)=(i d \otimes i d \otimes \varepsilon)(\Phi)=1 \otimes 1 .
$$

Together with a quasi-Hopf algebra $H=(H, \Delta, \varepsilon, \Phi, S, \alpha, \beta)$ we also have $H^{\text {op }}, H^{\text {cop }}$ and $H^{\mathrm{op} \text {,cop }}$ as quasi-Hopf algebras, where "op" means opposite multiplication and "cop" means opposite comultiplication. The quasi-Hopf structures are obtained by putting $\Phi_{\mathrm{op}}=\Phi^{-1}, \Phi_{\mathrm{cop}}=\left(\Phi^{-1}\right)^{321}, \Phi_{\mathrm{op}, \mathrm{cop}}=\Phi^{321}, S_{\mathrm{op}}=S_{\mathrm{cop}}=\left(S_{\mathrm{op}, \mathrm{cop}}\right)^{-1}=$ $S^{-1}, \alpha_{\mathrm{op}}=S^{-1}(\beta), \beta_{\mathrm{op}}=S^{-1}(\alpha), \alpha_{\mathrm{cop}}=S^{-1}(\alpha), \beta_{\mathrm{cop}}=S^{-1}(\beta), \alpha_{\mathrm{op}, \mathrm{cop}}=\beta$ and $\beta_{\text {op }, \mathrm{cop}}=\alpha$.

Recall that the definition of a quasi-Hopf algebra is "twist covariant" in the following sense. An invertible element $F \in H \otimes H$ is called a gauge transformation or twist if $(\varepsilon \otimes i d)(F)=(i d \otimes \varepsilon)(F)=1$. If $H$ is a quasi-Hopf algebra and $F=F^{1} \otimes F^{2} \in H \otimes H$ is a gauge transformation with inverse $F^{-1}=G^{1} \otimes G^{2}$, then we can define a new quasi-Hopf algebra $H_{F}$ by keeping the multiplication, unit, counit and antipode of $H$ and replacing the comultiplication, reassociator and the elements $\alpha$ and $\beta$ by

$$
\begin{aligned}
& \Delta_{F}(h)=F \Delta(h) F^{-1}, \\
& \Phi_{F}=(1 \otimes F)(i d \otimes \Delta)(F) \Phi(\Delta \otimes i d)\left(F^{-1}\right)\left(F^{-1} \otimes 1\right), \\
& \alpha_{F}=S\left(G^{1}\right) \alpha G^{2}, \beta_{F}=F^{1} \beta S\left(F^{2}\right) .
\end{aligned}
$$

It is well-known that the antipode of a Hopf algebra is an anti-coalgebra morphism. For a quasi-Hopf algebra, we have the following statement: there exists a gauge transformation $f \in H \otimes H$ such that

$$
f \Delta(S(h)) f^{-1}=(S \otimes S)\left(\Delta^{\mathrm{op}}(h)\right), \text { for all } h \in H,
$$

where $\Delta^{\mathrm{op}}(h)=h_{2} \otimes h_{1}$. The element $f$ can be computed explicitly. First set

$$
\begin{aligned}
& A^{1} \otimes A^{2} \otimes A^{3} \otimes A^{4}=(\Phi \otimes 1)(\Delta \otimes i d \otimes i d)\left(\Phi^{-1}\right), \\
& B^{1} \otimes B^{2} \otimes B^{3} \otimes B^{4}=(\Delta \otimes i d \otimes i d)(\Phi)\left(\Phi^{-1} \otimes 1\right) .
\end{aligned}
$$

Then define $\gamma, \delta \in H \otimes H$ by

$$
\gamma=S\left(A^{2}\right) \alpha A^{3} \otimes S\left(A^{1}\right) \alpha A^{4} \text { and } \delta=B^{1} \beta S\left(B^{4}\right) \otimes B^{2} \beta S\left(B^{3}\right) .
$$

Then $f$ and $f^{-1}$ are given by the formulae:

$$
\begin{aligned}
f & =(S \otimes S)\left(\Delta^{\mathrm{op}}\left(x^{1}\right)\right) \gamma \Delta\left(x^{2} \beta S\left(x^{3}\right)\right), \\
f^{-1} & =\Delta\left(S\left(x^{1}\right) \alpha x^{2}\right) \delta(S \otimes S)\left(\Delta^{\mathrm{op}}\left(x^{3}\right)\right) .
\end{aligned}
$$

Moreover, $f=f^{1} \otimes f^{2}$ and $g=g^{1} \otimes g^{2}$ satisfy the relations

$$
f \Delta(\alpha)=\gamma, \Delta(\beta) f^{-1}=\delta
$$

and (see [5])

$$
g^{1} S\left(g^{2} \alpha\right)=\beta, S\left(\beta f^{1}\right) f^{2}=\alpha .
$$


Furthermore the corresponding twisted reassociator (see (1.9)) is given by

$$
\Phi_{f}=(S \otimes S \otimes S)\left(X^{3} \otimes X^{2} \otimes X^{1}\right) .
$$

In a Hopf algebra $H$, we obviously have the identity $h_{1} \otimes h_{2} S\left(h_{3}\right)=h \otimes 1$, for all $h \in H$. We will need the generalization of this formula to the quasi-Hopf algebra setting. Following [12] and [13, we define

$$
\begin{aligned}
& p_{R}=p^{1} \otimes p^{2}=x^{1} \otimes x^{2} \beta S\left(x^{3}\right), \\
& q_{R}=q^{1} \otimes q^{2}=X^{1} \otimes S^{-1}\left(\alpha X^{3}\right) X^{2}, \\
& p_{L}=\tilde{p}^{1} \otimes \tilde{p}^{2}=X^{2} S^{-1}\left(X^{1} \beta\right) \otimes X^{3}, \\
& q_{L}=\tilde{q}^{1} \otimes \tilde{q}^{2}=S\left(x^{1}\right) \alpha x^{2} \otimes x^{3} .
\end{aligned}
$$

For all $h \in H$, we then have:

$$
\begin{aligned}
& \Delta\left(h_{1}\right) p_{R}\left[1 \otimes S\left(h_{2}\right)\right]=p_{R}[h \otimes 1], \\
& {\left[1 \otimes S^{-1}\left(h_{2}\right)\right] q_{R} \Delta\left(h_{1}\right)=(h \otimes 1) q_{R},} \\
& {\left[S\left(h_{1}\right) \otimes 1\right] q_{L} \Delta\left(h_{2}\right)=(1 \otimes h) q_{L},}
\end{aligned}
$$

and

$$
\begin{aligned}
& \Delta\left(q^{1}\right) p_{R}\left[1 \otimes S\left(q^{2}\right)\right]=1 \otimes 1, \\
& {\left[S\left(\tilde{p}^{1}\right) \otimes 1\right] q_{L} \Delta\left(\tilde{p}^{2}\right)=1 \otimes 1,} \\
& \Delta\left(\tilde{q}^{2}\right) p_{L}\left[S^{-1}\left(\tilde{q}^{1}\right) \otimes 1\right]=1 \otimes 1,
\end{aligned}
$$

$\Phi\left(\Delta \otimes i d_{H}\right)\left(p_{R}\right)\left(p_{R} \otimes i d_{H}\right)$

where $f=f^{1} \otimes f^{2}$ is the twist defined in (1.13) with its inverse $f^{-1}=g^{1} \otimes g^{2}$ defined in (1.14).

1.2. Quasitriangular quasi-Hopf algebras. A quasi-Hopf algebra $H$ is quasitriangular if there exists an element $R \in H \otimes H$ such that

$$
\begin{aligned}
(\Delta \otimes i d)(R) & =\Phi_{312} R_{13} \Phi_{132}^{-1} R_{23} \Phi, \\
(i d \otimes \Delta)(R) & =\Phi_{231}^{-1} R_{13} \Phi_{213} R_{12} \Phi^{-1}, \\
\Delta^{\mathrm{op}}(h) R & =R \Delta(h), \text { for all } h \in H, \\
(\varepsilon \otimes i d)(R) & =(i d \otimes \varepsilon)(R)=1 .
\end{aligned}
$$

Here we use the following notation. If $\sigma$ is a permutation of $\{1,2,3\}$, we set $\Phi_{\sigma(1) \sigma(2) \sigma(3)}=X^{\sigma^{-1}(1)} \otimes X^{\sigma^{-1}(2)} \otimes X^{\sigma^{-1}(3)}$, and $R_{i j}$ means $R$ acting non-trivially in the $i^{t h}$ and $j^{t h}$ positions of $H \otimes H \otimes H$.

In [5] it is shown that $R$ is invertible. The inverse of $R$ is given by

$$
R^{-1}=X^{1} \beta S\left(Y^{2} R^{1} x^{1} X^{2}\right) \alpha Y^{3} x^{3} X_{2}^{3} \otimes Y^{1} R^{2} x^{2} X_{1}^{3} .
$$

Furthermore, the element

$$
u=S\left(R^{2} p^{2}\right) \alpha R^{1} p^{1}
$$

(with $p_{R}=p^{1} \otimes p^{2}$ defined as in (1.18) ) satisfies $S^{2}(u)=u$, is invertible in $H$, and

$$
u^{-1}=X^{1} R^{2} p^{2} S\left(S\left(X^{2} R^{1} p^{1}\right) \alpha X^{3}\right),
$$

$$
\varepsilon(u)=1 \text { and } S^{2}(h)=u h u^{-1},
$$


for all $h \in H$. Consequently the antipode $S$ is bijective, so, as in the Hopf algebra case, the assumptions about invertibility of $R$ and bijectivity of $S$ can be dropped. Moreover, the $R$-matrix $R=R^{1} \otimes R^{2}$ satisfies the identities (see [1], 13, [5]):

$$
\begin{aligned}
& f_{21} R f^{-1}=(S \otimes S)(R), \\
& S\left(R^{2}\right) \alpha R^{1}=S(\alpha) u,
\end{aligned}
$$

where $f=f^{1} \otimes f^{2}$ is the twist defined in (1.13), and $f_{21}=f^{2} \otimes f^{1}$. In addition, a second formula for the inverse of $R$ is

$$
R^{-1}=\tilde{q}_{1}^{2} X^{2} R^{1} p^{1} \otimes \tilde{q}_{2}^{2} X^{3} S^{-1}\left(\tilde{q}^{1} X^{1} R^{2} p^{2}\right),
$$

where $p_{R}=p^{1} \otimes p^{2}$ and $q_{L}=\tilde{q}^{1} \otimes \tilde{q}^{2}$ are the elements defined by (1.18) and (1.21). Finally, recall that a quasi-Hopf algebra $(H, R)$ is called triangular if $R^{-1}=R_{21}$, where $R_{21}=R^{2} \otimes R^{1}$.

1.3. Monoidal categories. If $V$ is an object of a category $\mathcal{C}$, then the identity morphism $V \rightarrow V$ will also be denoted by $V$. The identity functor $\mathcal{C} \rightarrow \mathcal{C}$ will be denoted by $\mathcal{C}$.

A monoidal category $\mathcal{C}=(\mathcal{C}, \otimes, I, a, l, r)$ consists of a category $\mathcal{C}$, a functor $\otimes$ : $\mathcal{C} \times \mathcal{C} \rightarrow \mathcal{C}$, called the tensor product, an object $I \in \mathcal{C}$ called the unit object, and natural isomorphisms $a: \otimes \circ(\otimes \times \mathcal{C}) \rightarrow \otimes \circ(\mathcal{C} \times \otimes)$ (the associativity constraint), $l: \otimes \circ(I \times \mathcal{C}) \rightarrow \mathcal{C}$ (the left unit constraint) and $r: \otimes \circ(\mathcal{C} \times I) \rightarrow \mathcal{C}$ (the right unit constraint). $a$ has to satisfy the pentagon axiom, and $l$ and $r$ have to satisfy the triangle axiom. We refer to [14, XI.2] for a detailed discussion. In the sequel, we will identify $V \otimes I \cong I \cong I \otimes V$ using $l_{V}$ and $r_{V}$, for any object $V \in \mathcal{C}$.

A monoidal functor between two monoidal categories $\mathcal{C}$ and $\mathcal{D}$ is a triple $\left(F, \varphi_{0}, \varphi_{2}\right)$, where $F: \mathcal{C} \rightarrow \mathcal{D}$ is a functor, $\varphi_{0}: I \rightarrow F(I)$ is an isomorphism, and $\varphi_{2}(U, V)$ : $F(U) \otimes F(V) \rightarrow F(U \otimes V)$ is a family of natural isomorphisms in $\mathcal{D}$. $\varphi_{0}$ and $\varphi_{2}$ have to satisfy certain properties, see for example [14, XI.4].

If $H$ is a quasi-bialgebra, then the categories ${ }_{H} \mathcal{M}$ and $\mathcal{M}_{H}$ are monoidal categories. The associativity constraint on ${ }_{H} \mathcal{M}$ is the following: for $U, V, W \in{ }_{H} \mathcal{M}, a_{U, V, W}$ : $(U \otimes V) \otimes W \rightarrow U \otimes(V \otimes W)$ is given by

$$
a_{U, V, W}((u \otimes v) \otimes w)=X^{1} \cdot u \otimes\left(X^{2} \cdot v \otimes X^{3} \cdot w\right) .
$$

On $\mathcal{M}_{H}$, the associativity constraint is given by the formula

$$
a_{U, V, W}((u \otimes v) \otimes w)=u \cdot x^{1} \otimes\left(v \cdot x^{2} \otimes w \cdot x^{3}\right) .
$$

Let $V \in \mathcal{C} . V^{*} \in \mathcal{C}$ is called a left dual of $V$, if the functor $-\otimes V^{*}$ is the right dual of $-\otimes V$. This is equivalent to the existence of morphisms ev $V: V^{*} \otimes V \rightarrow I$ and coev : $I \rightarrow V \otimes V^{*}$ such that

$$
\begin{aligned}
& \left(V \otimes \mathrm{ev}_{V}\right) \circ a_{V, V^{*}, V} \circ\left(\operatorname{coev}_{V} \otimes V\right)=V, \\
& \left(\mathrm{ev}_{V} \otimes V^{*}\right) \circ a_{V^{*}, V, V^{*}}^{-1} \circ\left(V^{*} \otimes \operatorname{coev}_{V}\right)=V^{*},
\end{aligned}
$$

for all $V \in V \cdot{ }^{*} V \in \mathcal{C}$ is called a right dual of $V$ if $-\otimes{ }^{*} V$ is the left dual of $-\otimes V$. This is equivalent to the existence of morphisms $\mathrm{ev}_{V}^{\prime}: V \otimes{ }^{*} V \rightarrow I$ and $\operatorname{coev}_{V}^{\prime}: I \rightarrow{ }^{*} V \otimes V$ such that

$$
\begin{aligned}
& \left(\mathrm{ev}_{V}^{\prime} \otimes V\right) \circ a_{V,{ }^{*} V, V}^{-1} \circ\left(V^{*} \otimes \operatorname{coev}_{V}^{\prime}\right)=V, \\
& \left({ }^{*} V \otimes \mathrm{ev}_{V}^{\prime}\right) \circ a^{*} V, V,{ }^{*} V \circ\left(\operatorname{coev}_{V}^{\prime} \otimes V^{*}\right)=V^{*} \text {. }
\end{aligned}
$$

$\mathcal{C}$ is called a rigid monoidal category if every object of $\mathcal{C}$ has a left and right dual. The category ${ }_{H} \mathcal{M}^{\mathrm{fd}}$ of finite dimensional modules over a quasi-Hopf algebra $H$ is 
rigid. For $V \in{ }_{H} \mathcal{M}, V^{*}=\operatorname{Hom}(V, k)$, with left $H$-action $\langle h \cdot \varphi, v\rangle=\langle\varphi, S(h) v\rangle$. The evaluation and coevaluation are given by

$$
\operatorname{ev}_{V}(\varphi \otimes v)=\varphi(\alpha \cdot v), \quad \operatorname{coev}_{V}(1)=\beta \cdot v_{i} \otimes v^{i}
$$

where $\left\{v_{i}\right\}_{i}$ is a basis in $V$ with dual basis $\left\{v^{i}\right\}_{i}$. The right dual ${ }^{*} V$ of $V$ is the same dual vector space now equipped with the left $H$-module structure given by $\langle h \cdot \varphi, v\rangle=\left\langle\varphi, S^{-1}(h) v\right\rangle$, and with

$$
\operatorname{ev}_{V}^{\prime}(v \otimes \varphi)=\varphi\left(S^{-1}(\alpha) \cdot v\right), \operatorname{coev}_{V}^{\prime}(1)=v^{i} \otimes S^{-1}(\beta) \cdot v_{i} .
$$

The switch functor $\tau: \mathcal{C} \times \mathcal{C} \rightarrow \mathcal{C} \times \mathcal{C}$ is defined by $\tau(V, W)=(W, V)$. A prebraiding on a monoidal category is a natural transformation $c: \otimes \rightarrow \otimes \circ \tau$, satisfying the hexagon axioms (see for example [14 XIII.1]). A prebraiding $c$ is called a braiding if it is a natural isomorphism. Let $(H, R)$ be a quasitriangular quasi-bialgebra. We then have the following prebraiding $c$ on ${ }_{H} \mathcal{M}$ (see [14] or [16]):

$$
c_{U, V}(u \otimes v)=R^{2} \cdot v \otimes R^{1} \cdot u,
$$

which is a braiding if $H$ is a quasi-Hopf algebra.

1.4. Braided Hopf algebras. Let $\mathcal{C}$ be a braided monoidal category. We can define algebras, coalgebras, bialgebras and Hopf algebras, extending the classical definitions from 19 in the obvious way.

Thus, a bialgebra in $\mathcal{C}$ is $(B, m, \eta, \Delta, \varepsilon)$ where $B$ is an object in $\mathcal{C}$ and the morphism $m: B \otimes B \rightarrow B$ gives a multiplication that is associative up to the isomorphism $a$. Similarly for the coassociativity of the comultiplication $\Delta: B \rightarrow B \otimes B$. The identity in the algebra $B$ is expressed as usual by $\eta: I \rightarrow B$ such that $m \circ(\eta \otimes i d)=$ $m \circ(i d \otimes \eta)=i d$. The counit axiom is $(\varepsilon \otimes i d) \circ \Delta=(i d \otimes \varepsilon) \circ \Delta=i d$. In addition, $\Delta$ is required to be an algebra morphism where $B \otimes B$ has the multiplication $m_{B \otimes B}$, defined as the composition

$$
\begin{array}{ccccc}
(B \otimes B) \otimes(B \otimes B) & \underset{B \otimes c \otimes B}{\longrightarrow} & B \otimes(B \otimes(B \otimes B)) & \stackrel{B \otimes a^{-1}}{\longrightarrow} & B \otimes((B \otimes B) \otimes B) \\
\stackrel{a^{-1}}{\longrightarrow} & (B \otimes((B \otimes B) \otimes B) & \stackrel{B \otimes a}{\longrightarrow} & B \otimes(B \otimes(B \otimes B)) \\
\longrightarrow & (B \otimes(B \otimes B) & \stackrel{m \otimes m}{\longrightarrow} & B \otimes B .
\end{array}
$$

A Hopf algebra $B$ is a bialgebra with a morphism $S: B \rightarrow B$ in $\mathcal{C}$ (the antipode) satisfying the usual axioms $m \circ(S \otimes i d) \circ \Delta=\eta \circ \varepsilon=m \circ(i d \otimes S) \circ \Delta$.

For a braided monoidal category $\mathcal{C}$, let $\mathcal{C}^{\text {in }}$ be equal to $\mathcal{C}$ as a monoidal category, with the mirror-reversed braiding $\tilde{c}_{M, N}=c_{N, M}^{-1}$. For a Hopf algebra $B \in \mathcal{C}$ with bijective antipode, we define $B^{\text {op }}, B^{\text {cop }}$ and $B^{\text {op }, \text { cop }}$ by

$$
\begin{aligned}
& m_{B^{\mathrm{op}}}=m_{B} \circ c_{B, B}^{-1}, \Delta_{B^{\mathrm{op}}}=\Delta_{B}, S_{B^{\mathrm{op}}}=S_{B}^{-1}, \\
& m_{B^{\mathrm{cop}}}=m_{B}, \Delta_{B^{\mathrm{cop}}}=c_{B, B}^{-1} \circ \Delta_{B}, S_{B^{\mathrm{cop}}}=S_{B}^{-1}, \\
& m_{B^{\mathrm{op}, \mathrm{cop}}}=m_{B} \circ c_{B, B}, \Delta_{B^{\mathrm{op}, \mathrm{cop}}}=c_{B, B}^{-1} \circ \Delta_{B}, S_{B^{\mathrm{op}, \mathrm{cop}}}=S_{B},
\end{aligned}
$$

and the other structure morphisms remain the same as for $B$. It is well-known (see for example [2, 20] ) that $B^{\text {op }}$ and $B^{\text {cop }}$ are Hopf algebras in $\mathcal{C}^{\text {in }}$, and that $B^{\text {op,cop }}$ is a Hopf algebra in $\mathcal{C}$. 
1.5. Monoidal categories and the center construction. Let $\mathcal{C}$ be a monoidal category. Following [15], the weak left center $\mathcal{W}_{l}(\mathcal{C})$ is the category with the following objects and morphisms. An object is a couple $\left(V, s_{V,-}\right)$, with $V \in \mathcal{C}$ and $s_{V,-}: V \otimes-\rightarrow-\otimes V$ a natural transformation, satisfying the following condition, for all $X, Y \in \mathcal{C}$ :

$$
\left(X \otimes s_{V, Y}\right) \circ a_{X, V, Y} \circ\left(s_{V, X} \otimes Y\right)=a_{X, Y, V} \circ s_{V, X \otimes Y} \circ a_{V, X, Y},
$$

and such that $s_{V, I}$ is the composition of the natural isomorphisms $V \otimes I \cong V \cong$ $I \otimes V$. A morphism between $\left(V, s_{V,-}\right)$ and $\left(V^{\prime}, s_{V^{\prime},-}\right)$ consists of $\vartheta: V \rightarrow V^{\prime}$ in $\mathcal{C}$ such that

$$
(X \otimes \vartheta) \circ s_{V, X}=s_{V^{\prime}, X} \circ(\vartheta \otimes X) .
$$

$\mathcal{W}_{l}(\mathcal{C})$ is a prebraided monoidal category. The tensor product is

$$
\left(V, s_{V,-}\right) \otimes\left(V^{\prime}, s_{V^{\prime},-}\right)=\left(V \otimes V^{\prime}, s_{V \otimes V^{\prime},-}\right)
$$

with

$$
s_{V \otimes V^{\prime}, X}=a_{X, V, V^{\prime}} \circ\left(s_{V, X} \otimes V^{\prime}\right) \circ a_{V, X, V^{\prime}}^{-1} \circ\left(V \otimes s_{V^{\prime}, X}\right) \circ a_{V, V^{\prime}, X},
$$

and the unit is $(I, I)$. The prebraiding $c$ on $\mathcal{W}_{l}(\mathcal{C})$ is given by

$$
c_{V, V^{\prime}}=s_{V, V^{\prime}}:\left(V, s_{V,-}\right) \otimes\left(V^{\prime}, s_{V^{\prime},-}\right) \rightarrow\left(V^{\prime}, s_{V^{\prime},-}\right) \otimes\left(V, s_{V,-}\right) .
$$

The left center $\mathcal{Z}_{l}(\mathcal{C})$ is the full subcategory of $\mathcal{W}_{l}(\mathcal{C})$ consisting of objects $\left(V, s_{V,-}\right)$ with $s_{V,-}$ a natural isomorphism. $\mathcal{Z}_{l}(\mathcal{C})$ is a braided monoidal category. $\mathcal{Z}_{l}(\mathcal{C})^{\text {in }}$ will be our notation for the monoidal category $\mathcal{Z}_{l}(\mathcal{C})$, together with the inverse braiding $\tilde{c}$ given by $\tilde{c}_{V, V^{\prime}}=c_{V^{\prime}, V}^{-1}=s_{V^{\prime}, V}^{-1}$.

The weak right center $\mathcal{W}_{r}(\mathcal{C})$ and the right center $\mathcal{Z}_{r}(\mathcal{C})$ are introduced in a similar way. An object is a couple $\left(V, t_{-, V}\right)$, where $V \in \mathcal{C}$ and $t_{-, V}:-\otimes V \rightarrow V \otimes-$ is a family of natural transformations (resp. natural isomorphisms) such that $t_{-, I}$ is the natural isomorphism and

$$
a_{V, X, Y}^{-1} \circ t_{X \otimes Y, V} \circ a_{X, Y, V}^{-1}=\left(t_{X, V} \otimes Y\right) \circ a_{X, V, Y}^{-1} \circ\left(X \otimes t_{Y, V}\right),
$$

for all $X, Y \in \mathcal{C}$. A morphism between $\left(V, t_{-, V}\right)$ and $\left(V^{\prime}, t_{-, V^{\prime}}\right)$ consists of $\vartheta: V \rightarrow$ $V^{\prime}$ in $\mathcal{C}$ such that

$$
(\vartheta \otimes X) \circ t_{X, V}=t_{X, V^{\prime}} \circ(X \otimes \vartheta)
$$

for all $X \in \mathcal{C} . \mathcal{W}_{r}(\mathcal{C})$ is prebraided monoidal and $\mathcal{Z}_{r}(\mathcal{C})$ is braided monoidal. The unit is $(I, I)$ and the tensor product is now

$$
\left(V, t_{-, V}\right) \otimes\left(V^{\prime}, t_{-, V^{\prime}}\right)=\left(V \otimes V^{\prime}, t_{-, V \otimes V^{\prime}}\right)
$$

with

$$
t_{X, V \otimes V^{\prime}}=a_{V, V^{\prime}, X}^{-1} \circ\left(V \otimes t_{X, V^{\prime}}\right) \circ a_{V, X, V^{\prime}} \circ\left(t_{X, V} \otimes V^{\prime}\right) \circ a_{X, V, V^{\prime}}^{-1} .
$$

The braiding $d$ is given by

$$
d_{V, V^{\prime}}=t_{V, V^{\prime}}:\left(V, t_{-, V}\right) \otimes\left(V^{\prime}, t_{-, V^{\prime}}\right) \rightarrow\left(V^{\prime}, t_{-, V^{\prime}}\right) \otimes\left(V, t_{-, V}\right) .
$$

$\mathcal{Z}_{r}(\mathcal{C})^{\text {in }}$ is the monoidal category $\mathcal{Z}_{r}(\mathcal{C})$ with the inverse braiding $\tilde{d}$ given by $\tilde{d}_{V, V^{\prime}}=$ $d_{V^{\prime}, V}^{-1}=t_{V^{\prime}, V}^{-1}$.

For details in the case where $\mathcal{C}$ is a strict monoidal category, we refer to 14, Theorem XIII.4.2]. The results remain valid in the case of an arbitrary monoidal category, since every monoidal category is equivalent to a strict one. 
Proposition 1.1. Let $\mathcal{C}$ be a monoidal category. Then we have a braided isomorphism of braided monoidal categories $F: \mathcal{Z}_{l}(\mathcal{C}) \rightarrow \mathcal{Z}_{r}(\mathcal{C})^{\text {in }}$, given by

$$
F\left(V, s_{V,-}\right)=\left(V, s_{V,-}^{-1}\right) \text { and } F(\vartheta)=\vartheta .
$$

Proof. The proof is straighforward. Let us show that $F$ preserves the braiding. Applying $F$ to the braiding map

$$
c_{V, V^{\prime}}=s_{V, V^{\prime}}:\left(V, s_{V,-}\right) \otimes\left(V^{\prime}, s_{V^{\prime},-}\right) \rightarrow\left(V^{\prime}, s_{V^{\prime},-}\right) \otimes\left(V, s_{V,-}\right),
$$

we find

$$
F\left(s_{V, V^{\prime}}\right)=s_{V, V^{\prime}}:\left(V, s_{V,-}^{-1}\right) \otimes\left(V^{\prime}, s_{V^{\prime},-}^{-1}\right) \rightarrow\left(V^{\prime}, s_{V^{\prime},-}^{-1}\right) \otimes\left(V, s_{V,-}^{-1}\right) .
$$

Write $t_{-, V}=s_{V,-}^{-1}, t_{-, V^{\prime}}=s_{V^{\prime},-}^{-1}$. With notation as above, we find

$$
\tilde{d}_{V, V^{\prime}}=t_{V^{\prime}, V}^{-1}=s_{V, V^{\prime}},
$$

as needed.

Let $\mathcal{C}=(\mathcal{C}, \otimes, I, a, l, r)$ be a monoidal category. Then we have a second monoidal structure on $\mathcal{C}$, defined as follows:

$$
\overline{\mathcal{C}}=(\mathcal{C}, \bar{\otimes}=\otimes \circ \tau, I, \bar{a}, r, l)
$$

with $\tau: \mathcal{C} \times \mathcal{C} \rightarrow \mathcal{C} \times \mathcal{C}, \tau(V, W)=(W, V)$ and $\bar{a}$ defined by $\bar{a}_{V, W, X}=a_{X, W, V}^{-1}$. If $c$ is a (pre)braiding on $\mathcal{C}$, then $\bar{c}$, given by $\bar{c}_{V, W}=c_{W, V}$ is a (pre)braiding on $\overline{\mathcal{C}}$. The following result is then completely obvious.

Proposition 1.2. Let $\mathcal{C}$ be a monoidal category. Then

$$
\overline{\mathcal{W}_{l}(\mathcal{C})} \cong \mathcal{W}_{r}(\overline{\mathcal{C}}) ; \overline{\mathcal{W}_{r}(\mathcal{C})} \cong \mathcal{W}_{l}(\overline{\mathcal{C}}),
$$

as prebraided monoidal categories, and

$$
\overline{\mathcal{Z}_{l}(\mathcal{C})} \cong \mathcal{Z}_{r}(\overline{\mathcal{C}}) ; \overline{\mathcal{Z}_{r}(\mathcal{C})} \cong \mathcal{Z}_{l}(\overline{\mathcal{C}})
$$

as braided monoidal categories.

Proposition 1.3. Let $\mathcal{C}$ be a rigid monoidal category. Then the weak left (resp. right) center of $\mathcal{C}$ coincides with the left (resp. right) center of $\mathcal{C}$, and is a rigid braided monoidal category.

Proof. For details in the case where $\mathcal{C}$ is strict, we refer to [18, Lemma 7.2 and 7.3, Cor. 7.4]. The general case then follows from the fact that every monoidal category is equivalent to a strict one. For later use, we mention that for $(V, s) \in \mathcal{Z}_{l}(\mathcal{C})$, $\left(V, s_{V,-}\right)^{*}=\left(V^{*}, s_{V^{*},-}\right)$, with $s_{V^{*}, X}$ given by the following composition:

$$
\begin{aligned}
& s_{V^{*}, X}: V^{*} \otimes X \quad \stackrel{\left(V^{*} \otimes X\right) \otimes \operatorname{coev}_{V}}{\longrightarrow}\left(V^{*} \otimes X\right) \otimes\left(V \otimes V^{*}\right) \\
& \stackrel{a_{V^{*}, X, V \otimes V^{*}}}{\longrightarrow} \quad V^{*} \otimes\left(X \otimes\left(V \otimes V^{*}\right)\right) \\
& \stackrel{V^{*} \otimes a_{X, V, V^{*}}^{-1}}{\longrightarrow} \quad V^{*} \otimes\left((X \otimes V) \otimes V^{*}\right) \\
& \underset{V^{*} \otimes\left(s_{V, X}^{-1} \otimes V^{*}\right)}{\longrightarrow} \quad V^{*} \otimes\left((V \otimes X) \otimes V^{*}\right) \\
& \stackrel{V^{*} \otimes a_{V, X, V^{*}}}{\longrightarrow} \quad V^{*} \otimes\left(V \otimes\left(X \otimes V^{*}\right)\right) \\
& \underset{\mathrm{V}^{*}, V, X \otimes V^{*}}{\stackrel{a_{V} \otimes X \otimes V^{*}}{\longrightarrow}} \quad\left(V^{*} \otimes V\right) \otimes\left(X \otimes V^{*}\right) \\
& \stackrel{\mathrm{ev}_{V} \otimes X \otimes V^{*}}{\longrightarrow} \quad X \otimes V^{*} .
\end{aligned}
$$




\section{Yetter-Drinfeld modules over A QUASI-Hopf AlgeBra}

From [15], we recall the notion of left Yetter-Drinfeld module over a quasi-bialgebra. We also introduce right, left-right and right-left Yetter-Drinfeld modules. The aim of this Section is to study the relations between these four types of modules.

Definition 2.1. Let $H$ be a quasi-bialgebra, with reassociator $\Phi$. A left YetterDrinfeld module is a left $H$-module $M$ together with a $k$-linear map (called the left $H$-coaction)

$$
\lambda_{M}: M \rightarrow H \otimes M, \lambda_{M}(m)=m_{(-1)} \otimes m_{(0)}
$$

such that the following conditions hold, for all $h \in H$ and $m \in M$ :

$$
\begin{aligned}
& \quad X^{1} m_{(-1)} \otimes\left(X^{2} \cdot m_{(0)}\right)_{(-1)} X^{3} \otimes\left(X^{2} \cdot m_{(0)}\right)_{(0)} \\
& \quad=X^{1}\left(Y^{1} \cdot m\right)_{(-1)_{1}} Y^{2} \otimes X^{2}\left(Y^{1} \cdot m\right)_{(-1)_{2}} Y^{3} \otimes X^{3} \cdot\left(Y^{1} \cdot m\right)_{(0)}, \\
& \varepsilon\left(m_{(-1)}\right) m_{(0)}=m, \\
& h_{1} m_{(-1)} \otimes h_{2} \cdot m_{(0)}=\left(h_{1} \cdot m\right)_{(-1)} h_{2} \otimes\left(h_{1} \cdot m\right)_{(0)} .
\end{aligned}
$$

The category of left Yetter-Drinfeld modules and $k$-linear maps that preserve the $H$-action and $H$-coaction is denoted by ${ }_{H}^{H} \mathcal{Y} D$.

Let $H$ be a Hopf algebra. If $M$ is a left $H$-module and an $H$-comodule then (2.3) is equivalent to

$$
(h \cdot m)_{(-1)} \otimes(h \cdot m)_{(0)}=h_{1} m_{(-1)} S\left(h_{3}\right) \otimes h_{2} \cdot m_{(0)},
$$

for all $h \in H$ and $m \in M$. For quasi-Hopf algebras we have the following result.

Lemma 2.2. Let $H$ be a quasi-Hopf algebra, $M \in_{H} \mathcal{M}$, and $\lambda: M \rightarrow H \otimes M a$ $k$-linear map satisfying 2.12.2). Then (2.3) is equivalent to

$$
(h \cdot m)_{(-1)} \otimes(h \cdot m)_{(0)}=\left[q^{1} h_{1}\right]_{1}\left(p^{1} \cdot m\right)_{(-1)} p^{2} S\left(q^{2} h_{2}\right) \otimes\left[q^{1} h_{1}\right]_{2} \cdot\left(p^{1} \cdot m\right)_{(0)},
$$

for all $h \in H, m \in M$, where $p_{R}=p^{1} \otimes p^{2}$ and $q_{R}=q^{1} \otimes q^{2}$ are the elements defined in (1.18 1.19).

Proof. Suppose that (2.3) holds. For any $h \in H$ and $m \in M$ we compute that

$$
\begin{aligned}
(h \cdot m)_{(-1)} & \otimes(h \cdot m)_{(0)} \\
\frac{1.25}{=} & \left(q_{1}^{1} p^{1} h \cdot m\right)_{(-1)} q_{2}^{1} p^{2} S\left(q^{2}\right) \otimes\left(q_{1}^{1} p^{1} h \cdot m\right)_{(0)} \\
\frac{1.22}{=} & \left(\left[q^{1} h_{1}\right]_{1} p^{1} \cdot m\right)_{(-1)}\left[q^{1} h_{1}\right]_{2} p^{2} S\left(q^{2} h_{2}\right) \otimes\left(\left[q^{1} h_{1}\right]_{1} p^{1} \cdot m\right)_{(0)} \\
\frac{2.3}{=} & {\left[q^{1} h_{1}\right]_{1}\left(p^{1} \cdot m\right)_{(-1)} p^{2} S\left(q^{2} h_{2}\right) \otimes\left[q^{1} h_{1}\right]_{2} \cdot\left(p^{1} \cdot m\right)_{(0)} . }
\end{aligned}
$$

Conversely, assume that (2.4) holds; in particular we have that

$$
\lambda_{M}(m)=q_{1}^{1}\left(p^{1} \cdot m\right)_{(-1)} p^{2} S\left(q^{2}\right) \otimes q_{2}^{1} \cdot\left(p^{1} \cdot m\right)_{(0)}, \quad \forall m \in M .
$$

We then compute, for all $h \in H$ and $m \in M$ :

$$
\begin{array}{ll}
\left(h_{1} \cdot m\right)_{(-1)} & h_{2} \otimes\left(h_{1} \cdot m\right)_{(0)} \\
\frac{2.4}{=} & {\left[q^{1} h_{(1,1)}\right]_{1}\left(p^{1} \cdot m\right)_{(-1)} p^{2} S\left(q^{2} h_{(1,2)}\right) h_{2} \otimes\left[q^{1} h_{(1,1)}\right]_{2} \cdot\left(p^{1} \cdot m\right)_{(0)}} \\
\frac{1.23}{=} & h_{1} q_{1}^{1}\left(p^{1} \cdot m\right)_{(-1)} p^{2} S\left(q^{2}\right) \otimes h_{2} q_{2}^{1} \cdot\left(p^{1} \cdot m\right)_{(0)} \\
\frac{2.5}{=} & h_{1} m_{(-1)} \otimes h_{2} \cdot m_{(0)} .
\end{array}
$$


From [15], if $H$ is a quasi-bialgebra then the category of left Yetter-Drinfeld modules is isomorphic to the weak left center of the monoidal category $\mathcal{C}=:{ }_{H} \mathcal{M}$,

$$
\mathcal{W}_{l}\left({ }_{H} \mathcal{M}\right) \cong{ }_{H}^{H} \mathcal{Y} D
$$

The prebraided monoidal structure on $\mathcal{W}_{l}\left({ }_{H} \mathcal{M}\right)$ induces a prebraided monoidal structure on ${ }_{H}^{H} \mathcal{Y} D$. This structure is such that the forgetful functor ${ }_{H}^{H} \mathcal{Y} D \rightarrow{ }_{H} \mathcal{M}$ is monoidal. The coaction on the tensor product $M \otimes N$ of two Yetter-Drinfeld modules $M$ and $N$ is given by

$$
\lambda_{M \otimes N}(m \otimes n)=X^{1}\left(x^{1} Y^{1} \cdot m\right)_{(-1)} x^{2}\left(Y^{2} \cdot n\right)_{(-1)} Y^{3}
$$

$$
\otimes X^{2} \cdot\left(x^{1} Y^{1} \cdot m\right)_{(0)} \otimes X^{3} x^{3} \cdot\left(Y^{2} \cdot n\right)_{(0)},
$$

and the braiding is given by

$$
c_{M, N}(m \otimes n)=m_{(-1)} \cdot n \otimes m_{(0)} .
$$

Moreover, if $H$ is a quasi-Hopf algebra then $\mathcal{W}_{l}\left({ }_{H} \mathcal{M}\right)=\mathcal{Z}_{l}\left({ }_{H} \mathcal{M}\right) \cong{ }_{H}^{H} \mathcal{Y} D$, and the inverse of the braiding is given by (see [6]):

$$
c_{M, N}^{-1}(n \otimes m)=\tilde{q}_{1}^{2} X^{2} \cdot\left(p^{1} \cdot m\right)_{(0)} \otimes S^{-1}\left(\tilde{q}^{1} X^{1}\left(p^{1} \cdot m\right)_{(-1)} p^{2} S\left(\tilde{q}_{2}^{2} X^{3}\right)\right) \cdot n
$$

where $p_{R}=p^{1} \otimes p^{2}$ and $q_{L}=\tilde{q}^{1} \otimes \tilde{q}^{2}$ are the elements defined by (1.18) and (1.21), respectively.

We also introduce left-right, right-left and right Yetter-Drinfeld modules. More explicitly:

Definition 2.3. Let $H$ be a quasi-bialgebra, with reassociator $\Phi$.

1) A left-right Yetter-Drinfeld module is a left $H$-module $M$ together with a $k$-linear map (called the right $H$-coaction)

$$
\lambda_{M}: M \rightarrow M \otimes H, \rho_{M}(m)=m_{(0)} \otimes m_{(1)}
$$

such that the following conditions hold, for all $h \in H$ and $m \in M$ :

$$
\begin{aligned}
& \left(x^{2} \cdot m_{(0)}\right)_{(0)} \otimes\left(x^{2} \cdot m_{(0)}\right)_{(1)} x^{1} \otimes x^{3} m_{(1)} \\
& \quad=x^{1} \cdot\left(y^{3} \cdot m\right)_{(0)} \otimes x^{2}\left(y^{3} \cdot m\right)_{(1)_{1}} y^{1} \otimes x^{3}\left(y^{3} \cdot m\right)_{(1)_{2}} y^{2}, \\
& \varepsilon\left(m_{(1)}\right) m_{(0)}=m, \\
& h_{1} \cdot m_{(0)} \otimes h_{2} m_{(1)}=\left(h_{2} \cdot m\right)_{(0)} \otimes\left(h_{2} \cdot m\right)_{(1)} h_{1} .
\end{aligned}
$$

The category of left-right Yetter-Drinfeld modules is denoted by ${ }_{H} \mathcal{Y} D^{H}$.

2) A right-left Yetter-Drinfeld module is a right $H$-module $M$ together with a $k$ linear map (called the left $H$-coaction)

$$
\lambda_{M}: M \rightarrow H \otimes M, \lambda_{M}(m)=m_{(-1)} \otimes m_{(0)}
$$

such that the following conditions hold, for all $h \in H$ and $m \in M$ :

$$
\begin{aligned}
& m_{(-1)} x^{1} \otimes x^{3}\left(m_{(0)} \cdot x^{2}\right)_{(-1)} \otimes\left(m_{(0)} \cdot x^{2}\right)_{(0)} \\
& \quad=y^{2}\left(m \cdot y^{1}\right)_{(-1)_{1}} x^{1} \otimes y^{3}\left(m \cdot y^{1}\right)_{(-1)_{2}} x^{2} \otimes\left(m \cdot y^{1}\right)_{(0)} \cdot x^{3}, \\
& \varepsilon\left(m_{(-1)}\right) m_{(0)}=m, \\
& m_{(-1)} h_{1} \otimes m_{(0)} \cdot h_{2}=h_{2}\left(m \cdot h_{1}\right)_{(-1)} \otimes\left(m \cdot h_{1}\right)_{(0)} .
\end{aligned}
$$

The category of right-left Yetter-Drinfeld modules is denoted by ${ }^{H} \mathcal{Y} D_{H}$. 
3) A right Yetter-Drinfeld module is a right $H$-module $M$ together with a $k$-linear map (called the right $H$-coaction)

$$
\lambda_{M}: M \rightarrow M \otimes H, \rho_{M}(m)=m_{(0)} \otimes m_{(1)}
$$

such that the following conditions hold, for all $h \in H$ and $m \in M$ :

$$
\begin{aligned}
& \quad\left(m_{(0)} \cdot X^{2}\right)_{(0)} \otimes X^{1}\left(m_{(0)} \cdot X^{2}\right)_{(1)} \otimes m_{(1)} X^{3} \\
& \quad=\left(m \cdot Y^{3}\right)_{(0)} \cdot X^{1} \otimes Y^{1}\left(m \cdot Y^{3}\right)_{(1)_{1}} X^{2} \otimes Y^{2}\left(m \cdot Y^{3}\right)_{(1)_{2}} X^{3}, \\
& \begin{aligned}
\varepsilon\left(m_{(1)}\right) m_{(0)}=m, \\
m_{(0)} \cdot h_{1} \otimes m_{(1)} h_{2}=\left(m \cdot h_{2}\right)_{(0)} \otimes h_{1}\left(m \cdot h_{2}\right)_{(1)} .
\end{aligned}
\end{aligned}
$$

The category of right Yetter-Drinfeld modules is denoted by $\mathcal{Y} D_{H}^{H}$.

As in [15] the above definition of Yetter-Drinfeld modules was given using the center construction. More precisely:

Theorem 2.4. Let $H$ be a quasi-bialgebra. Then we have the following isomorphisms of categories:

$$
\mathcal{W}_{r}\left({ }_{H} \mathcal{M}\right) \cong{ }_{H} \mathcal{Y} \mathcal{D}^{H} ; \mathcal{W}_{r}\left(\mathcal{M}_{H}\right) \cong \mathcal{Y} \mathcal{D}_{H}^{H} ; \mathcal{W}_{l}\left(\mathcal{M}_{H}\right) \cong{ }^{H} \mathcal{Y} \mathcal{D}_{H}
$$

If $H$ is a quasi-Hopf algebra, then these three weak centers are equal to the centers.

Proof. (sketch) Take $\left(M, t_{-, M}\right) \in \mathcal{W}_{r}\left({ }_{H} \mathcal{M}\right)$, and consider $\rho_{M}=\left(\eta_{H} \otimes M\right) \circ t_{H, M}$ : $M \rightarrow M \otimes H$, that is,

$$
\rho_{M}(m)=m_{(0)} \otimes m_{(1)}=t_{H, M}(1 \otimes m) .
$$

$\rho_{M}$ determines $t_{-, M}$ completely: for $x \in X \in{ }_{H} \mathcal{M}$, consider the map

$$
f: H \rightarrow X, f(h)=h \cdot x
$$

in ${ }_{H} \mathcal{M}$. The naturality of $t_{-, M}$ entails that

$$
(M \otimes f) \circ t_{H, M}=t_{X, M} \circ(f \otimes M),
$$

and therefore

(2.18) $t_{X, M}(x \otimes m)=t_{X, M}((f \otimes M)(1 \otimes m))=m_{(0)} \otimes f\left(m_{(1)}\right)=m_{(0)} \otimes m_{(1)} \cdot x$. In particular, $m=t_{k, M}(1 \otimes m)=m_{(0)} \otimes m_{(1)} \cdot 1_{k}=\varepsilon\left(m_{(1)}\right) m_{(0)}$, so (2.10) holds. If we evaluate (1.56), with $X=Y=H$, at $1 \otimes 1 \otimes m$, we find (2.9). Finally,

$$
h \cdot t_{H, M}(1 \otimes m)=t_{H, M}\left(h_{1} \otimes h_{2} \cdot m\right)=\left(h_{2} \cdot m\right)_{(0)} \otimes\left(h_{2} \cdot m\right)_{(1)} h_{1},
$$

and

$$
h \cdot t_{H, M}(1 \otimes m)=h \cdot\left(m_{(0)} \otimes m_{(1)}\right)=h_{1} \cdot m_{(0)} \otimes h_{2} m_{(1)},
$$

proving (2.11), and we have shown that $\left(M, \rho_{M}\right)$ is a left-right Yetter-Drinfeld module.

Conversely, if $\left(M, \rho_{M}\right)$ is a left-right Yetter-Drinfeld module, $\left(M, t_{-, M}\right)$, with $t_{-, M}$ given by (2.18), is an object of $\mathcal{W}_{r}\left({ }_{H} \mathcal{M}\right)$. If $H$ has an antipode, then $t_{-, M}$ is invertible; the inverse is given by

$$
t_{N, M}^{-1}(m \otimes n)=q_{1}^{1} x^{1} S\left(q^{2} x^{3}\left(\tilde{p}^{2} \cdot m\right)_{(1)} \tilde{p}^{1}\right) \cdot n \otimes q_{2}^{1} x^{2} \cdot\left(\tilde{p}^{2} \cdot m\right)_{(0)}
$$

where $q_{R}=q^{1} \otimes q^{2}$ and $p_{L}=\tilde{p}^{1} \otimes \tilde{p}^{2}$ are the elements defined by (1.19) and (1.20), respectively.

The proof of the other two isomorphisms can be done in a similar way, we leave the details to the reader. 
The prebraided monoidal structure on $\mathcal{W}_{r}\left({ }_{H} \mathcal{M}\right)$ induces a monoidal structure on ${ }_{H} \mathcal{Y} D^{H}$. This structure is such that the forgetful functor ${ }_{H} \mathcal{Y} D^{H} \rightarrow_{H} \mathcal{M}$ is monoidal. Using (1.40) and (1.57), we find that the coaction on the tensor product $M \otimes N$ of two left-right Yetter-Drinfeld modules $M$ and $N$ is given by:

$$
\begin{aligned}
\rho_{M \otimes N}(m \otimes n)=x^{1} X^{1} \cdot\left(y^{2} \cdot m\right)_{(0)} \otimes x^{2} \cdot\left(X^{3} y^{3} \cdot n\right)_{(0)} \\
\otimes x^{3}\left(X^{3} y^{3} \cdot n\right)_{(1)} X^{2}\left(y^{2} \cdot m\right)_{(1)} y^{1},
\end{aligned}
$$

for all $m \in M, n \in N$. We have already observed that the functor forgetting the coaction is monoidal, so

$$
h \cdot(m \otimes n)=h_{1} \cdot m \otimes h_{2} \cdot n .
$$

The braiding $\mathfrak{c}$ can easily be deduced from (1.58) and (2.18): $\mathfrak{c}_{M, N}: M \otimes N \rightarrow N \otimes M$ is given by

$$
\mathfrak{c}_{M, N}(m \otimes n)=n_{(0)} \otimes n_{(1)} \cdot m,
$$

for $m \in M$ and $n \in N$. In the case when $H$ is a quasi-Hopf algebra, the inverse of the braiding is given by

$$
\mathfrak{c}_{M, N}^{-1}(n \otimes m)=q_{1}^{1} x^{1} S\left(q^{2} x^{3}\left(\tilde{p}^{2} \cdot n\right)_{(1)} \tilde{p}^{1}\right) \cdot m \otimes q_{2}^{1} x^{2} \cdot\left(\tilde{p}^{2} \cdot n\right)_{(0)} .
$$

${ }_{H} \mathcal{Y} D^{H^{\text {in }}}$ is the category with monoidal structure (2.2012.21) and the mirror reversed braiding $\tilde{\mathfrak{c}}_{M, N}=\mathfrak{c}_{N, M}^{-1}$.

For completeness' sake, let us also describe the prebraided monoidal structure on $\mathcal{Y} \mathcal{D}_{H}^{H}$ and ${ }^{H} \mathcal{Y} \mathcal{D}_{H}$. For $M, N \in \mathcal{Y} \mathcal{D}_{H}^{H}$, the coaction on $M \otimes N$ is given by the formula

$$
\begin{aligned}
& \rho(m \otimes n)=\left(m \cdot X^{2}\right)_{(0)} \cdot x^{1} Y^{1} \otimes\left(n \cdot X^{3} x^{3}\right)_{(0)} \cdot Y^{2} \\
& \otimes X^{1}\left(m \cdot X^{2}\right)_{(1)} x^{2}\left(n \cdot X^{3} x^{3}\right)_{(1)} Y^{3},
\end{aligned}
$$

and the $H$-action is

$$
(m \otimes n) \cdot h=m \cdot h_{1} \otimes n \cdot h_{2} .
$$

The braiding is the following:

$$
d_{M, N}(m \otimes n)=n_{(0)} \otimes m \cdot n_{(1)} .
$$

Now take $M, N \in{ }^{H} \mathcal{Y} \mathcal{D}_{H}$. The coaction on $M \otimes N$ is the following:

$$
\begin{aligned}
\lambda(m \otimes n)=x^{3}\left(n \cdot x^{2}\right)_{(-1)} & X^{2}\left(m \cdot x^{1} X^{1}\right)_{(-1)} y^{1} \\
& \left(m \cdot x^{1} X^{1}\right)_{(0)} \cdot y^{2} \otimes\left(n \cdot x^{2}\right)_{(0)} \cdot X^{3} y^{3},
\end{aligned}
$$

and the $H$-action is

$$
(m \otimes n) \cdot h=m \cdot h_{1} \otimes n \cdot h_{2} .
$$

The braiding is given by

$$
\mathfrak{d}_{M, N}(m \otimes n)=n \cdot m_{(-1)} \otimes m_{(0)} .
$$

Remark 2.5. Let $H$ be a quasi-bialgebra. If $\mathcal{C}={ }_{H} \mathcal{M}$, one can easily check that $\overline{\mathcal{C}} \cong{ }_{H^{c o p}} \mathcal{M}$. Then, by Proposition 1.2 we have $\mathcal{W}_{l}(\overline{\mathcal{C}}) \cong \overline{\mathcal{W}_{r}(\mathcal{C})}$, that is ${ }_{H^{c o p}}^{H^{c o p}} \mathcal{Y} D \cong$ $\bar{H} \mathcal{Y} D^{H}$ as prebraided monoidal categories; in an earlier version of this paper, we used this as a definition for left-right Yetter-Drinfeld modules in terms of left YetterDrinfeld modules.

Let $H$ be a quasi-bialgebra. We have that $H^{\text {op,cop }}$ is also a quasi-bialgebra. 
Proposition 2.6. We have an isomorphism of monoidal categories

$$
F: \overline{H^{\mathrm{op}, \mathrm{cop}} \mathcal{M}} \rightarrow \mathcal{M}_{H},
$$

given by $F(M)=M$ as a $k$-module, with right $H$-action $m \cdot h=h \cdot m$.

Proof. It is well-known and obvious that $F$ is an isomorphism of categories. So we only need to show that it preserves the monoidal structure. Let us first describe the monoidal structure on $H^{\mathrm{op}, \mathrm{cop}} \mathcal{M}$. The left $H^{\mathrm{op}, \mathrm{cop}}$-action on $N \otimes M$ is

$$
h \cdot(n \otimes m)=h_{2} \cdot n \otimes h_{1} \cdot m .
$$

The associativity constraint $a_{P, N, M}:(P \otimes N) \otimes M \rightarrow P \otimes(N \otimes M)$ is

$$
a_{P, N, M}((p \otimes n) \otimes m)=X^{3} \cdot p \otimes\left(X^{2} \cdot n \otimes X^{1} \cdot m\right) .
$$

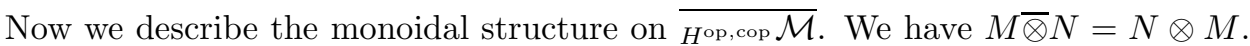
For $m \in M, n \in N$, we write

$$
m \bar{\otimes} n=n \otimes m \in M \bar{\otimes} N=N \otimes M .
$$

Then

$$
h \cdot(m \bar{\otimes} n)=h_{1} \cdot m \bar{\otimes} h_{2} \cdot n .
$$

The associativity constraint

$$
\bar{a}_{M, N, P}=a_{P, N, M}^{-1}:(M \bar{\otimes} N) \bar{\otimes} P=P \otimes(N \otimes M) \rightarrow M \bar{\otimes}(N \bar{\otimes} P)=(P \otimes N) \otimes M
$$

is given by

$$
\bar{a}_{M, N, P}((m \bar{\otimes} n) \bar{\otimes} p)=x^{1} \cdot m \bar{\otimes}\left(x^{2} \cdot n \bar{\otimes} x^{3} \cdot p\right) .
$$

It is then clear from (2.242.25) that $F$ preserves the monoidal structure.

An immediate consequence of Proposition 1.2. Theorem 2.4 and Proposition 2.6 is then the following.

Proposition 2.7. Let $H$ be a quasi-bialgebra. Then we have the following isomorphisms of prebraided monoidal categories, induced by the functor $F$ from Proposition 2.6];

$$
\mathcal{Y} \mathcal{D}_{H}^{H} \cong \overline{H^{\text {op }, \text { cop }} \mathcal{Y D}} \text { and }{ }^{H} \mathcal{Y} \mathcal{D}_{H} \cong \overline{H^{\text {op }, \text { cop }} \mathcal{Y} \mathcal{D}^{H^{\text {op }, \text { cop }}}} .
$$

Proof. We have the following isomorphisms of categories:

$$
\mathcal{Y} \mathcal{D}_{H}^{H} \cong \mathcal{W}_{r}\left(\mathcal{M}_{H}\right) \cong \mathcal{W}_{r}\left(\overline{H^{\mathrm{op}, \mathrm{cop}} \mathcal{M}}\right) \cong \overline{\mathcal{W}_{l}\left(H_{\mathrm{op}, \mathrm{cop}} \mathcal{M}\right)} \cong{ }_{H^{\mathrm{op}, \mathrm{cop}} \mathcal{Y D}} .
$$

Combining Proposition 1.1 and Theorem 2.4 we find the following result.

Theorem 2.8. Let $H$ be a quasi-Hopf algebra. Then we have an isomorphism of braided monoidal categories

$$
F:{ }_{H} \mathcal{Y} D^{H^{\text {in }}} \rightarrow{ }_{H}^{H} \mathcal{Y} D,
$$

defined as follows. For $M \in{ }_{H} \mathcal{Y} D^{H}, F(M)=M$ as a left $H$-module; the left $H$-coaction is given by

$$
\lambda_{M}(m)=m_{(-1)} \otimes m_{(0)}=q_{1}^{1} x^{1} S\left(q^{2} x^{3}\left(\tilde{p}^{2} \cdot m\right)_{(1)} \tilde{p}^{1}\right) \otimes q_{2}^{1} x^{2} \cdot\left(\tilde{p}^{2} \cdot m\right)_{(0)},
$$

for all $m \in M$, where $q_{R}=q^{1} \otimes q^{2}$ and $p_{L}=\tilde{p}^{1} \otimes \tilde{p}^{2}$ are the elements defined by (1.19) and [1.20), and $\rho_{M}(m)=m_{(0)} \otimes m_{(1)}$ is the right coaction of $H$ on $M$. The functor $F$ sends a morphism to itself. 
Proof. $F$ is nothing else then the composition of the isomorphisms

$$
{ }_{H} \mathcal{Y} D^{H^{\text {in }}} \rightarrow \mathcal{Z}_{r}\left({ }_{H} \mathcal{M}\right)^{\text {in }} \rightarrow \mathcal{Z}_{l}\left({ }_{H} \mathcal{M}\right) \rightarrow{ }_{H}^{H} \mathcal{Y} D .
$$

For $M \in{ }_{H} \mathcal{Y} D^{H^{\text {in }}}$, we compute that the corresponding left Yetter-Drinfeld module structure $\lambda_{M}$ on $M$ is the following:

$$
\begin{aligned}
\lambda_{M}(m) & =t_{H, M}^{-1}(m \otimes 1)=\tilde{\mathfrak{c}}_{M, H}(m \otimes 1)=\mathfrak{c}_{H, M}^{-1}(m \otimes 1) \\
& =q_{1}^{1} x^{1} S\left(q^{2} x^{3}\left(\tilde{p}^{2} \cdot m\right)_{(1)} \tilde{p}^{1}\right) \otimes q_{2}^{1} x^{2} \cdot\left(\tilde{p}^{2} \cdot m\right)_{(0)},
\end{aligned}
$$

as needed.

In the same way, we have the following result.

Theorem 2.9. Let $H$ be a quasi-Hopf algebra. Then the categories $\mathcal{Y} \mathcal{D}_{H}^{H}$ and ${ }^{H} \mathcal{Y} \mathcal{D}_{H}^{\text {in }}$ are isomorphic as braided monoidal categories.

We now recall some generalities about quasi-bialgebras. Let $H$ be a quasi-bialgebra, and $\mathfrak{F}=\mathfrak{F}^{1} \otimes \mathfrak{F}^{2} \in H \otimes H$ a twist with inverse $\mathfrak{F}^{-1}=\mathfrak{G}^{1} \otimes \mathfrak{G}^{2}$. Then we have an isomorphism of monoidal categories

$$
\Pi:{ }_{H} \mathcal{M} \rightarrow{ }_{H_{\mathfrak{F}}} \mathcal{M}
$$

$\Pi(M)=M$, with the same left $H$-action. If $H$ is a quasi-Hopf algebra, then we can consider the Drinfeld twist $f$ defined in (1.13). The antipode $S: H^{\mathrm{op}, \mathrm{cop}} \rightarrow$ $H_{f}$ is a quasi-Hopf algebra isomorphism, and therefore the monoidal categories $H^{\mathrm{op}, \mathrm{cop}} \mathcal{M}$ and ${ }_{H_{f}} \mathcal{M}$ are isomorphic. We have seen in Proposition 2.6 that ${ }_{H^{\mathrm{op}}, \mathrm{cop}} \mathcal{M}$ is isomorphic to $\overline{\mathcal{M}_{H}}$ as a monoidal category. We conclude that the monoidal categories ${ }_{H} \mathcal{M}$ and $\overline{\mathcal{M}_{H}}$ are isomorphic. Using Proposition 1.2 and Theorem 2.4 we find braided monoidal isomorphisms

$$
{ }_{H}^{H} \mathcal{Y} \mathcal{D} \cong \mathcal{Z}_{l}\left({ }_{H} \mathcal{M}\right) \cong \mathcal{Z}_{l}\left(\overline{\mathcal{M}_{H}}\right) \cong \overline{\mathcal{Z}_{r}\left(\mathcal{M}_{H}\right)} \cong \overline{\mathcal{Y} \mathcal{D}_{H}^{H}}
$$

and

$$
{ }_{H} \mathcal{Y} \mathcal{D}^{H} \cong \mathcal{Z}_{r}\left({ }_{H} \mathcal{M}\right) \cong \mathcal{Z}_{r}\left(\overline{\mathcal{M}_{H}}\right) \cong \overline{\mathcal{Z}_{l}\left(\mathcal{M}_{H}\right)} \cong \overline{{ }^{H} \mathcal{Y} \mathcal{D}_{H}}
$$

We summarize our results as follows:

Theorem 2.10. Let $H$ be a quasi-Hopf algebra. Then we have the following isomorphisms of braided monoidal categories:

$$
{ }_{H}^{H} \mathcal{Y D} \cong{ }_{H} \mathcal{Y} \mathcal{D}^{H}{ }^{\text {in }} \cong \overline{\mathcal{Y} \mathcal{D}_{H}^{H}} \cong{\overline{H_{\mathcal{Y D}}}}^{\text {in }} .
$$

The isomorphisms ${ }_{H}^{H} \mathcal{Y D} \cong \overline{\mathcal{Y} \mathcal{D}_{H}^{H}}$ and ${ }_{H} \mathcal{Y D}^{H} \cong \overline{{ }^{H} \mathcal{Y} \mathcal{D}_{H}}$ can be described explicitely. Let us compute the functor ${ }_{H}^{H} \mathcal{Y D} \rightarrow \overline{\mathcal{Y D}}{ }_{H}^{H}$.

We have a monoidal isomorphism $\Pi:{ }_{H} \mathcal{M} \rightarrow{ }_{H_{f}} \mathcal{M} ; \Pi(M)=M$ with the same left $H$-action. Denote the tensor product on $H_{f} \mathcal{M}$ by $\otimes^{f}$. For $M, N \in{ }_{H} \mathcal{M}$, the isomorphism $\psi: \Pi(M \otimes N) \rightarrow \Pi(M) \otimes^{f} \Pi(N)$ is given by

$$
\psi(m \otimes n)=f^{1} \cdot m \otimes^{f} f^{2} \cdot n .
$$

This isomorphism induces an isomorphism between the two left centers, hence between the categories ${ }_{H}^{H} \mathcal{Y D}$ and ${ }_{H_{f}}^{H_{f}} \mathcal{Y D}$. Take $\left(M, s_{M,-}\right) \in \mathcal{Z}_{l}\left({ }_{H} \mathcal{M}\right)$ and $\left(M, s_{M,-}^{f}\right)$ 
the corresponding object in $\mathcal{Z}_{l}\left({ }_{H_{f}} \mathcal{M}\right)$, and let $\lambda$ and $\lambda^{f}$ be the associated coactions. We then have the following commutative diagram

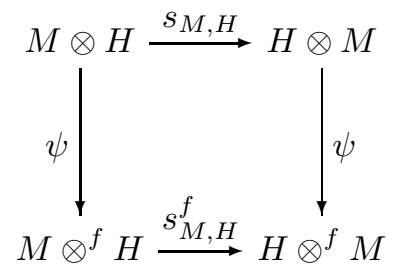

and we compute that

$\lambda^{f}(m)=s_{M, H}^{f}\left(m \otimes^{f} 1\right)=\psi\left(\left(s_{M, H}\left(g^{1} \cdot m \otimes g^{2}\right)\right)=f^{1}\left(g^{1} \cdot m\right)_{(-1)} g^{2} \otimes^{f} f^{2}\left(g^{1} \cdot m\right)_{(0)}\right.$.

The quasi-Hopf algebra isomorphism $S^{-1}: H_{f} \rightarrow H^{\mathrm{op}, \mathrm{cop}}$ induces an isomorphism of monoidal categories

$$
{ }_{H_{f}} \mathcal{M} \rightarrow{ }_{H^{\mathrm{op}, \operatorname{cop}} \mathcal{M} \cong \overline{\mathcal{M}_{H}}} .
$$

The image of $M$ is $M$ as a $k$-vector space, with right $H$-action given by $m \cdot h=$ $S(h) \cdot m$. Take $\left(M, s_{M,-}^{f}\right) \in \mathcal{Z}_{l}\left(H_{f} \mathcal{M}\right)$ and the corresponding object $\left(M, t_{-, M}\right) \in$ $\overline{\mathcal{Z}_{r}\left(\mathcal{M}_{H}\right)}$. Using similar arguments as above we can compute the right $H$-coaction $\rho$ on $M$ :

$$
\left.\rho_{M}(m)=f^{2} \cdot\left(g^{1} \cdot m\right)_{(0)} \otimes S^{-1}\left(f^{1}\left(g^{1} \cdot m\right)_{(-1)}\right) g^{2}\right) .
$$

We conclude that the braided isomorphism $K:{ }_{H}^{H} \mathcal{Y} D \rightarrow \overline{\mathcal{Y} \mathcal{D}_{H}^{H}}$ is defined as follows: $K(M)=M$, with

$$
\begin{aligned}
& m \cdot h=S(h) \cdot m, \\
& \rho_{M}(m)=f^{2} \cdot\left(g^{1} \cdot m\right)_{(0)} \otimes S^{-1}\left(f^{1}\left(g^{1} \cdot m\right)_{(-1)} g^{2}\right) .
\end{aligned}
$$

The inverse functor $K^{-1}$ can be computed in a similar way: $K^{-1}(M)=M$ with

$$
\begin{aligned}
& h \cdot m=m \cdot S^{-1}(h), \\
& \lambda_{M}(m)=g^{1} S\left(\left(m \cdot S^{-1}\left(f^{1}\right)\right)_{(1)}\right) f^{2} \otimes\left(m \cdot S^{-1}\left(f^{1}\right)\right)_{(0)} \cdot S^{-1}\left(g^{2}\right) .
\end{aligned}
$$

For completeness' sake, we also give the formulas for the braided monoidal isomorphism $G: \overline{{ }^{H} \mathcal{Y} \mathcal{D}_{H}} \rightarrow{ }_{H} \mathcal{Y} \mathcal{D}^{H} . G(M)=M$ with

$$
\begin{aligned}
& h \cdot m=m \cdot S^{-1}(h), \\
& \rho_{M}(m)=g^{1} \cdot\left(f^{2} \cdot m\right)_{(0)} \otimes g^{2} S\left(\left(f^{2} \cdot m\right)_{(-1)}\right) f^{1} .
\end{aligned}
$$

Conversely, $G^{-1}(M)=M$ with

$$
\begin{aligned}
& m \cdot h=S(h) \cdot m, \\
& \lambda_{M}(m)=S^{-1}\left(f^{2}\left(g^{2} \cdot m\right)_{(1)} g^{1}\right) \otimes f^{1} \cdot\left(g^{2} \cdot m\right)_{(0)} .
\end{aligned}
$$

\section{The RIGID BRAIDED CATEGory ${ }_{H}^{H} \mathcal{Y} D^{\mathrm{fd}}$}

It is well-known that the category of finite dimensional Yetter-Drinfeld modules over a coassociative Hopf algebra with invertible antipode is rigid. By Proposition 1.3 and since ${ }_{H} \mathcal{M}^{f d}$ is rigid the same result holds for the category of finite dimensional Yetter-Drinfeld modules over a quasi-Hopf algebra. We will give the explicit formulas in this Section. We first need a lemma. 
Lemma 3.1. Let $H$ be a quasi-Hopf algebra and $p_{R}=p^{1} \otimes p^{2}, q_{L}=\tilde{q}^{1} \otimes \tilde{q}^{2}=\tilde{Q}^{1} \otimes$ $\tilde{Q}^{2}$ and $f=f^{1} \otimes f^{2}$ the elements defined by (1.18), (1.21) and [1.13), respectively. Then the following relations hold:

$$
\begin{aligned}
& \tilde{q}^{1} X^{1} \otimes \tilde{q}_{1}^{2} X^{2} \otimes \tilde{q}_{2}^{2} X^{3}=S\left(x^{1}\right) \tilde{q}^{1} x_{1}^{2} \otimes \tilde{q}^{2} x_{2}^{2} \otimes x^{3}, \\
& S\left(p^{1}\right) \tilde{q}^{1} p_{1}^{2} S\left(\tilde{Q}^{2}\right)_{1} \otimes \tilde{Q}^{1} \tilde{q}^{2} p_{2}^{2} S\left(\tilde{Q}^{2}\right)_{2}=f .
\end{aligned}
$$

Proof. The equality (3.1) follows easily from (1.3), (1.5) and the definition of $q_{L}$. In order to prove (3.2), we denote by $\delta=\delta^{1} \otimes \delta^{2}$ the element defined in (1.12), and then compute that

$$
\begin{array}{ll}
S\left(p^{1}\right) \tilde{q}^{1} p_{1}^{2} S\left(\tilde{Q}^{2}\right)_{1} \otimes \tilde{Q}^{1} \tilde{q}^{2} p_{2}^{2} S\left(\tilde{Q}^{2}\right)_{2} \\
\frac{1.18}{=} & S\left(x^{1}\right) \tilde{q}^{1} x_{1}^{2} \beta_{1} S\left(\tilde{Q}^{2} x^{3}\right)_{1} \otimes \tilde{Q}^{1} \tilde{q}^{2} x_{2}^{2} \beta_{2} S\left(\tilde{Q}^{2} x^{3}\right)_{2} \\
\frac{1111.15}{1.111 .5} & S\left(x^{1}\right) \tilde{q}^{1} x_{1}^{2} \delta^{1} S\left(\tilde{Q}_{2}^{2} x_{2}^{3}\right) f^{1} \otimes \tilde{Q}^{1} \tilde{q}^{2} x_{2}^{2} \delta^{2} S\left(\tilde{Q}_{1}^{2} x_{1}^{3}\right) f^{2} \\
\frac{1.121 .31 .5}{=} & S\left(z^{1} x^{1}\right) \alpha z^{2} x_{1}^{2} y^{1} \beta S\left(\tilde{Q}_{2}^{2} x_{2}^{3} y_{2}^{3} X^{3}\right) f^{1} \otimes \tilde{Q}^{1} z^{3} x_{2}^{2} y^{2} X^{1} \beta S\left(\tilde{Q}_{1}^{2} x_{1}^{3} y_{1}^{3} X^{2}\right) f^{2} \\
\frac{1.31 .5}{=} & S\left(x^{1}\right) \alpha x^{2} \beta S\left(\tilde{Q}_{2}^{2} X^{3} x^{3}\right) f^{1} \otimes \tilde{Q}^{1} X^{1} \beta S\left(\tilde{Q}_{1}^{2} X^{2}\right) f^{2} \\
\frac{1.611 .20}{=} & S\left(\tilde{Q}_{2}^{2} \tilde{p}^{2}\right) f^{1} \otimes \tilde{Q}^{1} S\left(\tilde{Q}_{1}^{2} \tilde{p}^{1}\right) f^{1} \\
\frac{1.27}{=} & f^{1} \otimes f^{2}=f,
\end{array}
$$

as needed, and this finishes the proof.

Theorem 3.2. Let $H$ be a quasi-Hopf algebra. Then ${ }_{H}^{H} \mathcal{Y} D^{\mathrm{fd}}$ is a braided monoidal rigid category. For a finite dimensional left Yetter-Drinfeld module $M$ with basis $\left({ }_{i} m\right)_{i=\overline{1, n}}$ and corresponding dual basis $\left({ }^{i} m\right)_{i=\overline{1, n}}$, the left and right duals $M^{*}$ and ${ }^{*} M$ are equal to $\operatorname{Hom}(M, k)$ as a vector space, with the following $H$-action and $H$-coaction:

- For $M^{*}$ :

$$
\begin{aligned}
& \left(h \cdot m^{*}\right)(m)=m^{*}(S(h) \cdot m), \\
& \lambda_{M^{*}}\left(m^{*}\right)=m_{(-1)}^{*} \otimes m_{(0)}^{*} \\
& \quad=\left\langle m^{*}, f^{2} \cdot\left(g^{1} \cdot{ }_{i} m\right)_{(0)}\right\rangle S^{-1}\left(f^{1}\left(g^{1} \cdot{ }_{i} m\right)_{(-1)} g^{2}\right) \otimes{ }^{i} m .
\end{aligned}
$$

- For $^{*} M$ :

$$
\begin{aligned}
& \left(h \cdot{ }^{*} m\right)(m)={ }^{*} m\left(S^{-1}(h) \cdot m\right), \\
& \lambda_{* M}\left({ }^{*} m\right)={ }^{*} m_{(-1)} \otimes{ }^{*} m_{(0)} \\
& \quad=\left\langle{ }^{*} m, S^{-1}\left(f^{1}\right) \cdot\left(S^{-1}\left(g^{2}\right) \cdot{ }_{i} m\right)_{(0)}\right\rangle g^{1} S\left(\left(S^{-1}\left(g^{2}\right) \cdot{ }_{i} m\right)_{(-1)}\right) f^{2} \otimes{ }^{i} m,
\end{aligned}
$$

for all $h \in H, m^{*} \in M^{*},{ }^{*} m \in{ }^{*} M$ and $m \in M$. Here $f=f^{1} \otimes f^{2}$ is the twist defined by (1.13), with inverse $f^{-1}=g^{1} \otimes g^{2}$.

Proof. The left $H$-action on $M^{*}$ viewed as an element of ${ }_{H}^{H} \mathcal{Y} D$ is the same as the left $H$-action on $M^{*}$ viewed as an element of ${ }_{H} \mathcal{M}$. We compute the left $H$-coaction, using (1.59). By (2.8) in $\mathcal{Z}_{l}\left({ }_{H} \mathcal{M}\right) \cong{ }_{H}^{H} \mathcal{Y} D$ we have

$$
s_{V, X}^{-1}(v \otimes x)=\tilde{q}_{1}^{2} X^{2} \cdot\left(p^{1} \cdot x\right)_{(0)} \otimes S^{-1}\left(\tilde{q}^{1} X^{1}\left(p^{1} \cdot x\right)_{(-1)} p^{2} S\left(\tilde{q}_{2}^{2} X^{3}\right)\right) \cdot v
$$


for all $V, X \in{ }_{\tilde{Q}} \mathcal{M}, x \in X$ and $v \in V$. Now, if we denote by $P^{1} \otimes P^{2}$ another copy of $p_{R}$ and by $\tilde{Q}^{1} \otimes \tilde{Q}^{2}$ another copy of $q_{L}$ we then find

$$
\begin{aligned}
& \lambda\left(m^{*}\right)=s_{M^{*}, H}\left(m^{*} \otimes 1\right)=\left\langle x^{1} Z^{1} \cdot m^{*}, \alpha x^{2} Y^{1} \tilde{q}_{1}^{2} X^{2} \cdot\left(p^{1} y^{2} Z_{1}^{3} \beta \cdot{ }_{i} m\right)_{(0)}\right\rangle \\
& x_{1}^{3} Y^{2} \tilde{q}_{2}^{2} X^{3} S^{-1}\left(\tilde{q}^{1} X^{1}\left(p^{1} y^{2} Z_{1}^{3} \beta \cdot{ }_{i} m\right)_{(-1)} p^{2}\right) y^{1} Z^{2} \otimes x_{2}^{3} Y^{3} y^{3} Z_{2}^{3} \cdot{ }^{i} m \\
& \frac{1.511 .18}{=}\left\langle m^{*}, \tilde{Q}^{1} Y^{1} \tilde{q}_{1}^{2} X^{2} \cdot\left(p^{1} P^{2} S\left(\tilde{Q}_{2}^{2} Y^{3}\right) \cdot{ }_{i} m\right)_{(0)}\right\rangle \\
& \tilde{Q}_{1}^{2} Y^{2} \tilde{q}_{2}^{2} X^{3} S^{-1}\left(\tilde{q}^{1} X^{1}\left(p^{1} P^{2} S\left(\tilde{Q}_{2}^{2} Y^{3}\right) \cdot{ }_{i} m\right)_{(-1)} p^{2}\right) P^{1} \otimes{ }^{i} m \\
& \stackrel{3.12 .3}{=}\left\langle m^{*}, \tilde{Q}^{1} Y^{1} \tilde{q}^{2} \cdot\left(y_{1}^{2} p^{1} P^{2} S\left(\tilde{Q}_{2}^{2} Y^{3}\right) \cdot{ }_{i} m\right)_{(0)}\right\rangle \\
& \tilde{Q}_{1}^{2} Y^{2} y^{3} S^{-1}\left(\tilde{q}^{1}\left(y_{1}^{2} p^{1} P^{2} S\left(\tilde{Q}_{2}^{2} Y^{3}\right) \cdot{ }_{i} m\right)_{(-1)} y_{2}^{2} p^{2}\right) y^{1} P^{1} \otimes{ }^{i} m \\
& \frac{1.2822 .3}{=}\left\langle m^{*}, \tilde{Q}^{1} Y^{1} \tilde{q}^{2} x_{(2,2)}^{1} p_{2}^{2} \cdot\left(g^{1} S\left(\tilde{Q}_{2}^{2} Y^{3} x^{3}\right) \cdot{ }_{i} m\right)_{(0)}\right\rangle \\
& \tilde{Q}_{1}^{2} Y^{2} S^{-1}\left(\tilde{q}^{1} x_{(2,1)}^{1} p_{1}^{2} \cdot\left(g^{1} S\left(\tilde{Q}_{2}^{2} Y^{3} x^{3}\right) \cdot{ }_{i} m\right)_{(-1)} g^{2} S\left(x^{2}\right)\right) x_{1}^{1} p^{1} \otimes{ }^{i} m \\
& \underset{=}{1.241 .11}\left\langle m^{*}, \tilde{Q}^{1} \tilde{q}^{2} p_{2}^{2} \cdot\left(S\left(\tilde{Q}^{2}\right)_{1} g^{1} \cdot{ }_{i} m\right)_{(0)}\right\rangle \\
& S^{-1}\left(\tilde{q}^{1} p_{1}^{2} \cdot\left(S\left(\tilde{Q}^{2}\right)_{1} g^{1} \cdot{ }_{i} m\right)_{(-1)} S\left(\tilde{Q}^{2}\right)_{2} g^{2}\right) p^{1} \otimes{ }^{i} m \\
& \stackrel{2.313 .2}{=}\left\langle m^{*}, f^{2} \cdot\left(g^{1} \cdot{ }_{i} m\right)_{(0)}\right\rangle S^{-1}\left(f^{1} \cdot\left(g^{1} \cdot{ }_{i} m\right)_{(-1)} g^{2}\right) \otimes{ }^{i} m \text {, }
\end{aligned}
$$

as claimed. The structure on ${ }^{*} M$ can be computed in a similar way, we leave the details to the reader.

\section{The CANONiCAL isomorphisms in ${ }_{H}^{H} \mathcal{Y} D^{\text {fd }}$}

If $\mathcal{C}$ is a braided rigid category then, for any two objects $M, N \in \mathcal{C}$, there exist two canonical isomorphisms in $\mathcal{C}: M \cong M^{* *}$ and $(M \otimes N)^{*} \cong M^{*} \otimes N^{*}$. In this section our goal is to compute explicitly the above isomorphisms in the particular case $\mathcal{C}={ }_{H}^{H} \mathcal{Y} D^{f . d}$. Then we will specialize them for the category of finite dimensional modules over a quasitriangular quasi-Hopf algebra.

Let $\mathcal{C}$ be a rigid monoidal category and $M, N$ objects of $\mathcal{C}$. If $\nu: M \rightarrow N$ is a morphism in $\mathcal{C}$, following [14] we can define the transposes of $\nu$ as being the compositions; the left and right unit constraints are treated as the identity.

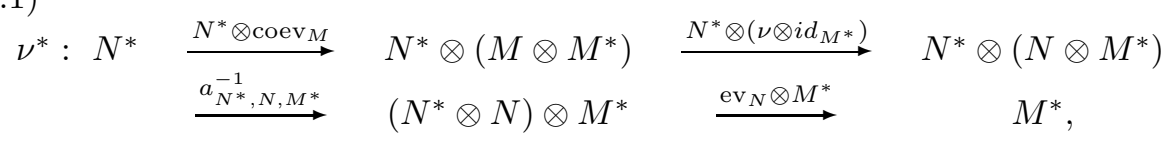

and

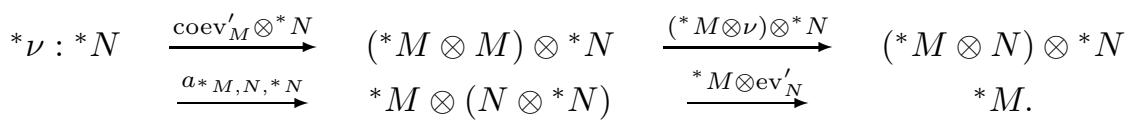

Since the functors $-\otimes V$ and $-\otimes *\left(V^{*}\right)$ are left duals of $-\otimes V^{*}$, they are naturally isomorphic, so we have an isomorphism $\theta_{M}: V \cong *\left(V^{*}\right)$, and this isomorphism is natural in $M$. In the same way, we have a natural isomorphism $\theta_{M}^{\prime}: V \cong\left({ }^{*} V\right)^{*}$. 
D. BULACU, S. CAENEPEEL, AND F. PANAITE

Let us describe $\theta_{M}$ and its invers explicitly, we refer to [16 for details.

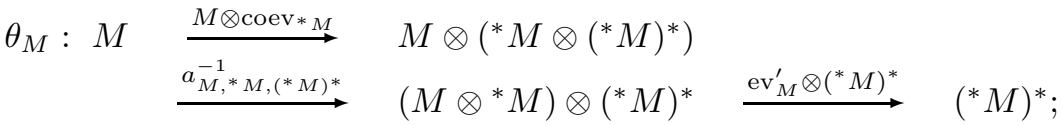

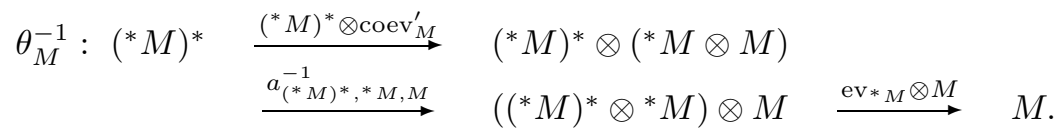

We also have a natural isomorphism $\Theta_{M}: M^{*} \rightarrow{ }^{*} M$. this can be described as follows, see 2] for details.

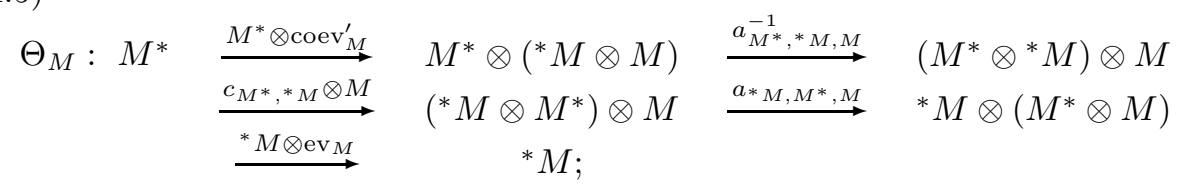

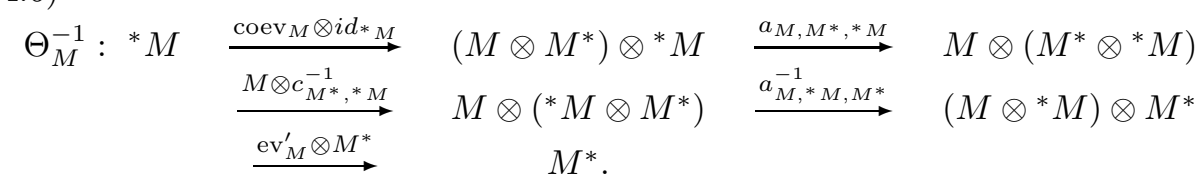

Thus the functors $(-)^{*}$ and ${ }^{*}(-)$ are naturally isomorphic, and we conclude that

$$
M^{* *}=\left(M^{*}\right)^{*} \cong{ }^{*}\left(M^{*}\right) \cong M \cong\left({ }^{*} M\right)^{*} \cong{ }^{*}\left({ }^{*} M\right)={ }^{* *} M .
$$

We will apply these results in the particular case where $\mathcal{C}={ }_{H}^{H} \mathcal{Y} D^{\mathrm{fd}}$.

1) The maps $\nu^{*}$ and ${ }^{*} \nu$ coincide with the usual transposed map of $\nu$. Indeed, by (4.1) and (1.6), we have that

$$
\begin{aligned}
\nu^{*}\left(n^{*}\right) & =\left\langle x^{1} \cdot n^{*}, \alpha x^{2} \beta \cdot \nu\left({ }_{i} n\right)\right\rangle x^{3} \cdot{ }^{i} n \\
& =\left\langle n^{*}, S\left(x^{1}\right) \alpha x^{2} \beta S\left(x^{3}\right) \cdot \nu\left({ }_{i} n\right)\right\rangle^{i} n=n^{*} \circ \nu,
\end{aligned}
$$

where $\left({ }_{i} n\right)_{i=\overline{1, t}}$ is a basis of $N$ and $\left({ }^{i} n\right)_{i=\overline{1, t}}$ its dual basis. A similar computation shows that ${ }^{*} \nu\left({ }^{*} n\right)={ }^{*} n \circ \nu$ for any ${ }^{*} n \in{ }^{*} N$.

2) It is not hard to see that the map $\theta_{M}$ defined by 4.3) is given by

$$
\theta_{M}(m)=\left\langle{ }^{i} m, m\right\rangle^{* i} m
$$

for all $m \in M$, where, if $\left({ }_{i} m\right)_{i=1, n}$ is a basis of $M$ with dual basis $\left({ }^{i} m\right)_{i=\overline{1, n}}$ in $M^{*}$, then ${ }^{* i} m$ is the image of ${ }_{i} m$ under the canonical map $M \rightarrow M^{* *}$. Moreover, the morphism $\theta_{M}^{\prime}$ is defined by the same formula (4.7) as $\theta_{M}$. A straightforward but tedious computation proves that the morphism $\Theta_{M}: M^{*} \rightarrow{ }^{*} M$ defined by (4.5) is given by

$$
\Theta_{M}\left(m^{*}\right)=\left\langle m^{*}, S\left(p^{1}\right) f^{2} \cdot\left(g^{1} \cdot{ }_{j} m\right)_{(0)}\right\rangle
$$

$$
\left\langle{ }^{j} m, S\left(q^{2}\right) S^{-1}\left(q^{1} S^{-1}\left(f^{1}\left(g^{1} \cdot{ }_{j} m\right)_{(-1)} g^{2}\right) p^{2}\right) \cdot{ }_{i} m\right\rangle^{i} m,
$$

for all $m^{*} \in M^{*}$. The inverse map $\Theta_{M}^{-1}:{ }^{*} M \rightarrow M^{*}$ is given by (see (4.6)

$$
\Theta_{M}^{-1}\left({ }^{*} m\right)=\left\langle{ }^{*} m, V^{2} \cdot{ }_{j} m_{(0)}\right\rangle\left\langle{ }^{j} m, \beta S\left(V^{1}{ }_{j} m_{(-1)}\right) \cdot{ }_{i} m\right\rangle^{i} m,
$$

for any ${ }^{*} m \in{ }^{*} M$, where

$$
V=V^{1} \otimes V^{2}=S^{-1}\left(f^{2} p^{2}\right) \otimes S^{-1}\left(f^{1} p^{1}\right) .
$$


Therefore, we obtain the following result.

Proposition 4.1. Let $H$ be a quasi-Hopf algebra and $M, N \in{ }_{H}^{H} \mathcal{Y} D^{\mathrm{fd}}$. Then ${ }^{r} \Gamma_{M}=\theta_{M}^{\prime-1} \circ \Theta_{M^{*}}: M^{* *} \rightarrow M$ is an isomorphism of Yetter-Drinfeld modules. Explicitly, ${ }^{r} \Gamma_{M}$ is given by

(4.11) ${ }^{r} \Gamma_{M}\left(m^{* *}\right)=\left\langle m^{* *},{ }^{i} m\right\rangle q^{1} S^{-2}\left(\left(S^{2}\left(p^{1}\right) \cdot{ }_{i} m\right)_{(-1)}^{\mathcal{F}}\right) p^{2} S^{2}\left(q^{2}\right) \cdot\left(S^{2}\left(p^{1}\right) \cdot{ }_{i} m\right)_{(0)}^{\mathcal{F}}$

for all $m^{* *} \in M^{* *}$, where

$$
\mathcal{F}=\mathcal{F}^{1} \otimes \mathcal{F}^{2}=S\left(g^{2}\right) f^{1} \otimes S\left(g^{1}\right) f^{2},
$$

and where, in general, if $\mathfrak{F}=\mathfrak{F}^{1} \otimes \mathfrak{F}^{2}$ is a twist on $H$ and $M \in{ }_{H}^{H} \mathcal{Y} D$ then we denote

$$
\lambda_{M}^{\mathfrak{F}}(m)=m_{(-1)}^{\mathfrak{F}} \otimes m_{(0)}^{\mathfrak{F}}:=\mathfrak{F}^{1}\left(\mathfrak{G}^{1} \cdot m\right)_{(-1)} \mathfrak{G}^{2} \otimes \mathfrak{F}^{2} \cdot\left(\mathfrak{G}^{1} \cdot m\right)_{(0)},
$$

where $\mathfrak{G}=\mathfrak{G}^{1} \otimes \mathfrak{G}^{2}=\mathfrak{F}^{-1}$.

The inverse of ${ }^{r} \Gamma_{M}$ is given by ${ }^{r} \Gamma_{M}^{-1}=\Theta_{M^{*}}^{-1} \circ \theta_{M}^{\prime}$, that is,

$$
{ }^{r} \Gamma_{M}^{-1}(m)=\left\langle{ }^{i} m, S\left(\left(p^{1} \cdot m\right)_{(-1)} p^{2}\right) \alpha \cdot\left(p^{1} \cdot m\right)_{(0)}\right\rangle^{* i} m .
$$

Similarly, ${ }^{l} \Gamma_{M}:=\theta_{M}^{-1} \circ \Theta_{*_{M}}^{-1}:{ }^{* *} M \rightarrow M$ provides an isomorphism of YetterDrinfeld modules. Explicitly, we have that

(4.14) ${ }^{l} \Gamma_{M}\left({ }^{* *} m\right)=\left\langle{ }^{* *} m,{ }^{i} m\right\rangle V^{1} g^{1} S\left(\left(S^{-1}\left(V^{2} g^{2}\right) \cdot{ }_{i} m\right)_{(-1)}\right) \alpha \cdot\left(S^{-1}\left(V^{2} g^{2}\right) \cdot{ }_{i} m\right)_{(0)}$, for all ${ }^{* *} m \in{ }^{* *} M$. The inverse of ${ }^{l} \Gamma_{M}$ is given by

$$
{ }^{l} \Gamma_{M}^{-1}(m)=\left\langle{ }^{i} m, S^{-2}\left(q^{1}\left(p^{1} \cdot m\right)_{(-1)} p^{2}\right) q^{2} \cdot\left(p^{1} \cdot m\right)_{(0)}\right\rangle^{* i} m .
$$

Proof. Straightforward, we leave the details to the reader.

Now, we will focus on the second isomorphism. Let $\mathcal{C}$ be a rigid braided monoidal category and $M, N \in \mathcal{C}$. By [2] there exist natural isomorphisms

$$
\begin{gathered}
\sigma_{M, N}^{*}: M^{*} \otimes N^{*} \rightarrow(M \otimes N)^{*} \\
{ }^{*} \sigma_{M, N}:{ }^{*} M \otimes{ }^{*} N \rightarrow{ }^{*}(M \otimes N) .
\end{gathered}
$$

In fact, $\sigma_{M, N}^{*}=\phi_{N, M}^{*} \circ c_{N^{*}, M^{*}}^{-1}$, where $\phi_{N, M}^{*}: N^{*} \otimes M^{*} \rightarrow(M \otimes N)^{*}$ is the composition

$$
\begin{aligned}
\phi_{N, M}^{*}= & \mathrm{ev}_{N} \otimes(M \otimes N)^{*} \circ\left(N^{*} \otimes\left(\operatorname{ev}_{M} \otimes N\right)\right) \otimes(M \otimes N)^{*} \\
& \circ N^{*} \otimes a_{M^{*}, M, N}^{-1} \otimes(M \otimes N)^{*} \circ a_{N^{*}, M^{*}, M \otimes N} \otimes(M \otimes N)^{*} \\
& \circ a_{N^{*} \otimes M^{*}, M \otimes N,(M \otimes N)^{*}}^{-1} \circ N^{*} \otimes M^{*} \otimes \operatorname{coev}_{M \otimes N}
\end{aligned}
$$

with inverse

$$
\begin{aligned}
\phi_{N, M}^{*-1}= & \left(\operatorname{ev}_{M \otimes N} \otimes N^{*}\right) \otimes M^{*} \circ a_{(M \otimes N)^{*}, M \otimes N, N^{*}}^{-1} \otimes M^{*} \\
& \circ\left((M \otimes N)^{*} \otimes a_{M, N, N^{*}}^{-1}\right) \otimes M^{*} \circ\left((M \otimes N)^{*} \otimes\left(M \otimes \operatorname{coev}_{N}\right)\right) \otimes M^{*} \\
(4.17) \quad & \circ a_{(M \otimes N)^{*}, M, M^{*}}^{-1} \circ(M \otimes N)^{*} \otimes \operatorname{coev}_{M} .
\end{aligned}
$$

In a similar way, we can define ${ }^{*} \phi_{N, M}:{ }^{*} N \otimes{ }^{*} M \rightarrow{ }^{*}(M \otimes N)$ and its inverse ${ }^{*} \phi_{N, M}^{-1}$; then we put ${ }^{*} \sigma_{M, N}={ }^{*} \phi_{N, M} \circ c^{-1} N,{ }^{*} M$. 
Proposition 4.2. Let $H$ be a quasi-Hopf algebra and $M, N \in{ }_{H}^{H} \mathcal{Y} D^{\mathrm{fd}}$. Consider $\left\{{ }_{i} m\right\}_{i=\overline{1, s}}$ and $\left\{{ }^{i} m\right\}_{i=\overline{1, s}}$ be dual bases in $M$ and $M^{*}$, and $\left\{{ }_{j} n\right\}_{j=\overline{1, t}}$ and $\left\{{ }^{j} n\right\}_{j=\overline{1, t}}$ dual bases in $N$ and $N^{*}$. Then the map $\sigma_{M, N}^{*}: M^{*} \otimes N^{*} \rightarrow(M \otimes N)^{*}$ defined by

$$
\begin{aligned}
& \sigma_{M, N}^{*}\left(m^{*} \otimes n^{*}\right)(m \otimes n) \\
& \quad=\left\langle m^{*}, f^{2} \tilde{q}_{2}^{2} X^{3} S^{-1}\left(\tilde{q}^{1} X^{1}\left(p^{1} \cdot n\right)_{(-1)} p^{2}\right) \cdot m\right\rangle\left\langle n^{*}, f^{1} \tilde{q}_{1}^{2} X^{2} \cdot\left(p^{1} \cdot n\right)_{(0)}\right\rangle
\end{aligned}
$$

is an isomorphism of Yetter-Drinfeld modules. Here $p_{R}=p^{1} \otimes p^{2}$ and $q_{L}=\tilde{q}^{1} \otimes \tilde{q}^{2}$ are the elements defined in (1.18) and 1.21). The inverse of $\sigma_{M, N}^{*}$ is given by

$$
\sigma_{M, N}^{*-1}(\mu)=\left\langle\mu,\left(g^{1} \cdot{ }_{j} n\right)_{(-1)} g^{2} \cdot{ }_{i} m \otimes\left(g^{1} \cdot{ }_{j} n\right)_{(0)}\right\rangle^{i} m \otimes{ }^{j} n .
$$

In a similar way, the map ${ }^{*} \sigma_{M, N}:{ }^{*} M \otimes{ }^{*} N \rightarrow{ }^{*}(M \otimes N)$ defined by

$$
\begin{gathered}
{ }^{*} \sigma_{M, N}\left({ }^{*} m \otimes{ }^{*} n\right)(m \otimes n)=\left\langle{ }^{*} m, S^{-1}\left(f^{2} q^{1}\left(x^{1} \tilde{p}^{1} S^{-1}\left(f^{1}\right) \cdot n\right)_{(-1)} x^{2} \tilde{p}_{1}^{2}\right) \cdot m\right\rangle \\
\left\langle{ }^{*} n, S^{-1}\left(x^{3} \tilde{p}_{2}^{2}\right) q^{2} \cdot\left(x^{1} \tilde{p}^{1} S^{-1}\left(f^{1}\right) \cdot n\right)_{(0)}\right\rangle
\end{gathered}
$$

is an isomorphism of Yetter-Drinfeld modules; $q_{R}=q^{1} \otimes q^{2}$ and $p_{L}=\tilde{p}^{1} \otimes \tilde{p}^{2}$ are the elements defined in (1.19) and (1.20). The inverse of ${ }^{*} \sigma_{M, N}$ is given by

$$
{ }^{*} \sigma_{M, N}^{-1}(\nu)=\left\langle\nu,\left(S^{-1}\left(g^{2}\right) \cdot{ }_{j} n\right)_{(-1)} S^{-1}\left(g^{1}\right) \cdot{ }_{i} m \otimes\left(S^{-1}\left(g^{2}\right) \cdot{ }_{j} n\right)(0)\right\rangle^{i} m \otimes{ }^{j} n .
$$

Proof. We will show that $\sigma_{M, N}^{*}=\phi_{N, M^{*}}^{*} c_{N^{*}, M^{*}}^{-1}$, where $\phi_{N, M}^{*}$ is given by (4.16). We first calculate $\phi_{N, M}^{*}$. As before, we write $q_{R}=q^{1} \otimes q^{2}$ and $p_{R}=p^{1} \otimes p^{2}=P^{1} \otimes P^{2}$, and then compute for all $n^{*} \in N^{*}, m^{*} \in M^{*}, m \in M$ and $n \in N$ that:

$$
\begin{array}{cc}
\phi_{N, M}^{*}\left(n^{*} \otimes m^{*}\right)(m \otimes n) \\
\frac{4.16}{=} & \left\langle m^{*}, S\left(x^{1} X^{2} y_{2}^{1}\right) \alpha x^{2}\left(X^{3} y^{2} \beta S\left(y^{3}\right)\right)_{1} \cdot m\right\rangle \\
& \left\langle n^{*}, S\left(X^{1} y_{1}^{1}\right) \alpha x^{3}\left(X^{3} y^{2} \beta S\left(y^{3}\right)\right)_{2} \cdot n\right\rangle \\
\frac{1.12}{=} & \left\langle m^{*}, S\left(y_{2}^{1}\right) \gamma^{1}\left(y^{2} \beta S\left(y^{3}\right)\right)_{1} \cdot m\right\rangle\left\langle n^{*}, S\left(y_{1}^{1}\right) \gamma^{2}\left(y^{2} \beta S\left(y^{3}\right)\right)_{2} \cdot n\right\rangle \\
\frac{1.111 .18}{=} & \left\langle m^{*}, f^{1} S\left(p^{1}\right)_{1} g^{1} \gamma^{1} p_{1}^{2} \cdot m\right\rangle\left\langle n^{*}, f^{2} S\left(p^{1}\right)_{2} g^{2} \gamma^{2} p_{2}^{2} \cdot n\right\rangle \\
\frac{1.15}{=} & \left\langle m^{*}, f^{1}\left(S\left(p^{1}\right) \alpha p^{2}\right)_{1} \cdot m\right\rangle\left\langle n^{*}, f^{2}\left(S\left(p^{1}\right) \alpha p^{2}\right)_{2} \cdot n\right\rangle \\
\frac{1.181 .6}{=} & \left\langle m^{*}, f^{1} \cdot m\right\rangle\left\langle n^{*}, f^{2} \cdot n\right\rangle .
\end{array}
$$

Using (4.17) or by a direct computation it is easy to see that

$$
\phi_{N, M}^{*-1}(\mu)=\left\langle\mu, g^{1} \cdot{ }_{i} m \otimes g^{2} \cdot{ }_{j} n\right\rangle^{j} n \otimes{ }^{i} m
$$

for all $\mu \in(M \otimes N)^{*}$. Also, it is not hard to see that (1.9) and (1.17) imply that

$$
q^{1} g_{1}^{1} \otimes S\left(q^{2} g_{2}^{1}\right) g^{2}=S\left(X^{3}\right) f^{1} \otimes S\left(X^{1} \beta S\left(X^{2}\right) f^{2}\right) .
$$

The same relations and (1.16) imply that

$$
S\left(p^{2} f^{1}\right) F^{1} f_{1}^{2} \otimes S\left(p^{1}\right) F^{2} f_{2}^{2}=q_{L} .
$$

Using (4.23), the axioms of a quasi-Hopf algebra, again (1.9) and (1.17), and finally (1.26) we obtain the following relation:

$$
\begin{gathered}
q^{1}\left[g^{1} S\left(\tilde{q}_{1}^{2} X^{2}\right) f^{2}\right]_{1} \otimes S\left(q^{2}\left[g^{1} S\left(\tilde{q}_{1}^{2} X^{2}\right) f^{2}\right]_{2}\right) g^{2} S\left(\tilde{q}^{1} X^{1}\right) \otimes S\left(\tilde{q}^{2} X^{3}\right) f^{1} \\
=f^{2} F_{2}^{2} x^{2} \otimes S\left(F^{2} x^{3}\right) \otimes f^{1} F_{1}^{1} x^{1}
\end{gathered}
$$


Let $f=F^{1} \otimes F^{2}=\mathbf{F}^{1} \otimes \mathbf{F}^{2}$ and $p_{R}=P^{1} \otimes P^{2}$ be other copies of $f$ and $p_{R}$. For $m^{*} \in M^{*}, n^{*} \in N^{*}, m \in M$ and $n \in N$ we calculate:

$$
\begin{array}{ll}
\left(\phi_{N, M}^{*} \circ c_{N^{*}, M^{*}}^{-1}\right)\left(m^{*} \otimes n^{*}\right)(m \otimes n) \\
\frac{2.83 .4}{=} & \left\langle m^{*}, \tilde{q}^{1} X^{1} S^{-1}\left(F^{1}\left(g^{1} S\left(\tilde{q}_{1}^{2} X^{2}\right) f^{2} \cdot n\right)_{(-1)} g^{2}\right) p^{2} S\left(\tilde{q}_{2}^{2} X^{3}\right) f^{1} \cdot m\right\rangle \\
& \left\langle n^{*}, S\left(p^{1}\right) F^{2} \cdot\left(g^{1} S\left(\tilde{q}_{1}^{2} X^{2}\right) f^{2} \cdot n\right)_{(0)}\right\rangle \\
\frac{2.44 .25}{=} & \left\langle m^{*}, S^{-1}\left(\mathbf{F}^{1} f_{1}^{2} F_{(2,1)}^{1} x_{1}^{2}\left(P^{1} \cdot n\right)_{(-1)} P^{2} S\left(F^{2} x^{3}\right)\right) p^{2} f^{1} F_{1}^{1} x^{1} \cdot m\right\rangle \\
& \left\langle n^{*}, S\left(p^{1}\right) \mathbf{F}^{2} f_{2}^{2} F_{(2,2)}^{1} x_{2}^{2} \cdot\left(P^{1} \cdot n\right)_{(0)}\right\rangle \\
\frac{4.2514 .24}{=} & \left\langle m^{*}, S^{-1}\left(\tilde{q}^{1} F_{(2,1)}^{1} x_{1}^{2}\left(P^{1} \cdot n\right)_{(-1)} P^{2} S\left(F^{2} x^{3}\right)\right) F_{1}^{1} x^{1}\right\rangle \\
& \left\langle n^{*}, F_{2}^{1} \tilde{q}^{2} x_{2}^{2} \cdot\left(P^{1} \cdot n\right)_{(0)}\right\rangle \\
\frac{1.2411 .3}{=} & \left\langle m^{*}, F^{2} \tilde{q}_{2}^{2} X^{3} S^{-1}\left(\tilde{q}^{1} X^{1}\left(P^{1} \cdot n\right)_{(-1)} P^{2}\right) \cdot m\right\rangle \\
& \left\langle n^{*}, F^{1} \tilde{q}_{1}^{2} X^{2} \cdot\left(P^{1} \cdot n\right)_{(0)}\right\rangle \\
\frac{4.18}{=} & \sigma_{M, N}^{*}\left(m^{*} \otimes n^{*}\right)(m \otimes n) .
\end{array}
$$

Obviously, the inverse of $\sigma_{M, N}^{*}$ is $\sigma_{M, N}^{*-1}=c_{N^{*}, M^{*}} \circ \phi_{N, M}^{*-1}$. By (4.22) it follows that $\sigma_{M, N}^{*-1}$ is defined by the formula given in (4.19). The assertion concerning the morphism ${ }^{*} \sigma_{M, N}$ can be proved in a similar way. We only notice that

$$
{ }^{*} \phi_{N, M}\left({ }^{*} n \otimes{ }^{*} m\right)(m \otimes n)=\left\langle{ }^{*} m, S^{-1}\left(f^{2}\right) \cdot m\right\rangle\left\langle{ }^{*} n, S^{-1}\left(f^{1}\right) \cdot n\right\rangle
$$

for all ${ }^{*} m \in{ }^{*} M,{ }^{*} n \in{ }^{*} N, m \in M$ and $n \in N$.

Let $(H, R)$ be a quasitriangular quasi-Hopf algebra. Then we have a monoidal fuctor $\mathbb{F}:{ }_{H} \mathcal{M} \rightarrow{ }_{H}^{H} \mathcal{Y} D$ which acts as the identity on objects and morphisms. For $M \in{ }_{H} \mathcal{M}, \mathbb{F}(M)=M$ as a left $H$-module, and with left $H$-coaction given by

$$
\lambda_{M}(m)=R^{2} \otimes R^{1} \cdot m,
$$

for all $m \in M$. Moreover, this functor sends algebras, coalgebras, bialgebras etc. in ${ }_{H} \mathcal{M}$ to the corresponding objects in ${ }_{H}^{H} \mathcal{Y} D$ (see [6] Proposition 2.4]).

Corollary 4.3. (8, Lemma 3.2]). Let $H$ be a quasitriangular quasi-Hopf algebra and $M$ a finite dimensional left $H$-module. Then $M \cong M^{* *}$ and $M \cong{ }^{* *} M$ as $H$-modules.

Proof. We have seen above that $M$ can be viewed as an object in ${ }_{H}^{H} \mathcal{Y} D$, so $M \cong M^{* *}$ and $M \cong{ }^{* *} M$ as Yetter-Drinfeld modules, cf. Proposition 4.1 Thus, $M \cong M^{* *}$ and $M \cong{ }^{* *} M$ as $H$-modules. We will write down explicitly these isomorphisms. We have that ${ }^{r} \Gamma_{M}: M^{* *} \rightarrow M$ is given by

$$
{ }^{r} \Gamma_{M}\left(m^{* *}\right)=\left\langle m^{* *},{ }^{i} m\right\rangle u^{-1} \cdot{ }_{i} m,
$$

with inverse

$$
{ }^{r} \Gamma_{M}^{-1}(m)=\left\langle{ }^{i} m, u \cdot m\right\rangle^{* i} m,
$$

for all $m^{* *} \in M^{* *}$ and $m \in M$, which is equivalent to ${ }^{r} \Gamma_{M}^{-1}(m)(\varphi)=\varphi(u \cdot m)$ for all $\varphi \in M^{*}$ and $m \in M$. In this way we recover the isomorphism $\hat{\psi}: M \rightarrow M^{* *}$ given in 8 .

Similarly, ${ }^{l} \Gamma_{M}:{ }^{* *} M \rightarrow M$ is given by

$$
{ }^{l} \Gamma\left({ }^{* *} m\right)=\left\langle{ }^{* *} m,{ }^{i} m\right\rangle u \cdot{ }_{i} m
$$


for all ${ }^{* *} m \in{ }^{* *} M$, with inverse

$$
{ }^{l} \Gamma_{M}^{-1}(m)=\left\langle{ }^{i} m, u^{-1} \cdot m\right\rangle^{* i} m
$$

for all $m \in M$.

Remark 4.4. The element $u$ plays a central role in the theory of quasitriangular Hopf algebras. It is therefore natural to try to generalize its properties to the quasi-Hopf algebra setting. The major problem in [5] was to find the inverse of the element $u$ defined in [1. Now, if we forget the definitions of $u$ and $u^{-1}$ in the quasi-Hopf case, combining Corollary 4.3 with the similar result in the Hopf case we will obtain in a natural way the definitions for $u$ and its inverse $u^{-1}$.

Corollary 4.5. (8 Lemma 3.3]). Let $(H, R)$ be a quasitriangular quasi-Hopf algebra and $M, N$ two finite dimensional left $H$-modules. Then $M^{*} \otimes N^{*} \cong(M \otimes N)^{*}$ and ${ }^{*} M \otimes{ }^{*} N \cong{ }^{*}(M \otimes N)$ as $H$-modules.

Proof. We regard $M, N$ as objects in ${ }_{H}^{H} \mathcal{Y} D^{\mathrm{fd}}$ via the functor $\mathbb{F}$ defined in (4.26). Thus, by Proposition 4.2 we obtain that $M^{*} \otimes N^{*} \cong(M \otimes N)^{*}$ and ${ }^{*} M \otimes{ }^{*} N \cong$ $*(M \otimes N)$ as Yetter-Drinfeld modules, so they are isomorphic also as $H$-modules. Moreover, (1.39) implies that the isomorphism $\sigma_{M, N}^{*}: M^{*} \otimes N^{*} \rightarrow(M \otimes N)^{*}$ defined in (4.18) is given by

$$
\sigma_{M, N}^{*}\left(m^{*} \otimes n^{*}\right)(m \otimes n)=\left\langle m^{*}, f^{2} \bar{R}^{2} \cdot m\right\rangle\left\langle n^{*}, f^{1} \bar{R}^{1} \cdot n\right\rangle
$$

for all $m^{*} \in M^{*}, n^{*} \in N^{*}, m \in M, n \in N$, where $R^{-1}:=\sum \bar{R}^{1} \otimes \bar{R}^{2}$ is the inverse of the $R$-matrix $R$. Note that it is just the isomorphism $\mu_{M, N}$ defined in [8]. Also, 1.33) implies that the isomorphism ${ }^{*} \sigma_{M, N}:{ }^{*} M \otimes{ }^{*} N \rightarrow{ }^{*}(M \otimes N)$ defined by (4.20) is given by

$$
{ }^{*} \sigma_{M, N}\left({ }^{*} m \otimes{ }^{*} n\right)(m \otimes n)=\left\langle{ }^{*} m, S^{-1}\left(f^{2} \bar{R}^{2}\right) \cdot m\right\rangle\left\langle{ }^{*} n, S^{-1}\left(f^{1} \bar{R}^{1}\right) \cdot n\right\rangle,
$$

for all ${ }^{*} m \in{ }^{*} M,{ }^{*} n \in{ }^{*} N, m \in M$ and $n \in N$. Finally, it is not hard to see that

$$
\sigma_{M, N}^{*-1}(\mu)=\left\langle\mu, R^{2} g^{2} \cdot{ }_{i} m \otimes R^{1} g^{1} \cdot{ }_{j} n\right\rangle^{i} m \otimes{ }^{j} n
$$

for all $\mu \in(M \otimes N)^{*}$, and

$$
\begin{array}{ccc}
{ }^{*} \sigma_{M, N}^{-1}(\nu) & \frac{4.204 .26}{=} & \left\langle\nu, R^{2} S^{-1}\left(g^{1}\right) \cdot{ }_{i} m \otimes R^{1} S^{-1}\left(g^{2}\right) \cdot{ }_{j} n\right\rangle^{i} m \otimes{ }^{j} n \\
& \frac{1.37}{=} & \left\langle\nu, S^{-1}\left(R^{2} g^{2}\right) \cdot{ }_{i} m \otimes S^{-1}\left(R^{1} g^{1}\right) \cdot{ }_{j} n\right\rangle^{i} m \otimes{ }^{j} n
\end{array}
$$

for any $\nu \in{ }^{*}(M \otimes N)$, completing the proof.

Remark 4.6. Continuing the ideas of Remark 4.4 we notice that the above Corollary suggests the two formulae (1.33) and (1.39) for the inverse of the $R$-matrix $R$, and, also, the formula (1.37). All these formulae where first proved by Hauser and Nill [13] in the case that $(H, R)$ is a finite dimensional quasitriangular quasi-Hopf algebra. They also used the bijectivity of the antipode. 


\section{Applications}

Let $B$ be a Hopf algebra in a braided rigid category $\mathcal{C}$. Then $B^{*}$ and ${ }^{*} B$ are also Hopf algebras in $\mathcal{C}$, see 2] 20]. The structure maps on $B^{*}$ are the following ones:

$$
\begin{array}{ll}
m_{B^{*}}: & B^{*} \otimes B^{*} \stackrel{\sigma_{B, B}^{*}}{\longrightarrow}(B \otimes B)^{*} \stackrel{\Delta_{B}^{*}}{\longrightarrow} B^{*}, \\
\Delta_{B^{*}}: & B^{*} \stackrel{m_{B}^{*}}{\longrightarrow}(B \otimes B)^{*} \stackrel{\sigma_{B, B}^{*-1}}{\longrightarrow} B^{*} \otimes B^{*}, \\
S_{B^{*}}= & S_{B}^{*}, u_{B^{*}}=\varepsilon_{B}^{*}, \varepsilon_{B^{*}}=u_{B}^{*} .
\end{array}
$$

The structure maps on the left dual can be obtained in a similar way.

We can consider algebras, coalgebras, bialgebras and Hopf algebras in the braided category ${ }_{H}^{H} \mathcal{Y} D$. More precisely, an algebra in ${ }_{H}^{H} \mathcal{Y} D$ is an object $B \in{ }_{H}^{H} \mathcal{Y} D$ with the additional structure:

- $B$ is a left $H$-module algebra, i.e. $B$ has a multiplication and a unit $1_{B}$ satisfying the conditions:

$$
\begin{aligned}
(a b) c & =\left(X^{1} \cdot a\right)\left[\left(X^{2} \cdot b\right)\left(X^{3} \cdot c\right)\right], \\
h \cdot(a b) & =\left(h_{1} \cdot a\right)\left(h_{2} \cdot b\right), \\
h \cdot 1_{B} & =\varepsilon(h) 1_{B},
\end{aligned}
$$

for all $a, b, c \in B$ and $h \in H$.

- $B$ is a quasi-comodule algebra, that is, the multiplication $m$ and the unit $\eta$ of $B$ intertwine the $H$-coaction $\lambda_{B}$. By (2.6), this means:

$$
\begin{aligned}
\lambda_{B}\left(b b^{\prime}\right) & =X^{1}\left(x^{1} Y^{1} \cdot b\right)_{(-1)} x^{2}\left(Y^{2} \cdot b^{\prime}\right)_{(-1)} Y^{3} \\
& \otimes\left[X^{2} \cdot\left(x^{1} Y^{1} \cdot b\right)_{(0)}\right]\left[X^{3} x^{3} \cdot\left(Y^{2} \cdot b^{\prime}\right)_{(0)}\right],
\end{aligned}
$$

for all $b, b^{\prime} \in B$, and

$$
\lambda_{B}\left(1_{B}\right)=1_{H} \otimes 1_{B} .
$$

A coalgebra in ${ }_{H}^{H} \mathcal{Y} D$ is an object $B$ with

- $B$ is a left $H$-module coalgebra, i.e. $B$ has a comultiplication $\Delta_{B}: B \rightarrow$ $B \otimes B$ (we will denote $\underline{\Delta}(b)=b_{\underline{1}} \otimes b_{2}$ ) and a usual counit $\underline{\varepsilon}_{B}$ such that:

$$
\begin{aligned}
& X^{1} \cdot b_{(\underline{1}, \underline{1})} \otimes X^{2} \cdot b_{(\underline{1}, \underline{2})} \otimes X^{3} \cdot b_{\underline{2}}=b_{\underline{1}} \otimes b_{(\underline{2}, \underline{1})} \otimes b_{(\underline{2}, \underline{2})}, \\
& \underline{\Delta}_{B}(h \cdot b)=h_{1} \cdot b_{\underline{1}} \otimes h_{2} \cdot b_{\underline{2}}, \quad \underline{\varepsilon}_{B}(h \cdot b)=\varepsilon(h) \underline{\varepsilon}_{B}(b),
\end{aligned}
$$

for all $h \in H, b \in B$, where we adopt for the quasi-coassociativity of $\underline{\Delta}_{B}$ the same notations as in the Section 1

- $B$ is a quasi-comodule coalgebra, i.e. the comultiplication $\underline{\Delta}_{B}$ and the counit $\underline{\varepsilon}_{B}$ intertwine the $H$-coaction $\lambda_{B}$. Explicitly, for all $b \in B$ we must have that:

$$
\begin{gathered}
b_{(-1)} \otimes b_{(0)_{1}} \otimes b_{(0)_{\underline{2}}}=X^{1}\left(x^{1} Y^{1} \cdot b_{\underline{1}}\right)_{(-1)} x^{2}\left(Y^{2} \cdot b_{\underline{2}}\right)_{(-1)} Y^{3} \otimes \\
X^{2} \cdot\left(x^{1} Y^{1} \cdot b_{\underline{1}}\right)_{(0)} \otimes X^{3} x^{3} \cdot\left(Y^{2} \cdot b_{\underline{2}}\right)_{(0)},
\end{gathered}
$$

and

$$
\underline{\varepsilon}_{B}\left(b_{(0)}\right) b_{(-1)}=\underline{\varepsilon}_{B}(b) 1 .
$$


An object $B \in{ }_{H}^{H} \mathcal{Y} D$ is a bialgebra if it is an algebra and a coalgebra in ${ }_{H}^{H} \mathcal{Y} D$ such that $\underline{\Delta}_{B}$ is an algebra morphism, i.e. $\underline{\Delta}_{B}\left(1_{B}\right)=1_{B} \otimes 1_{B}$ and, by (1.49) and (2.7), for all $b, b^{\prime} \in B$ we have that:

$$
\begin{array}{r}
\Delta_{B}\left(b b^{\prime}\right)=\left[y^{1} X^{1} \cdot b_{1}\right]\left[y^{2} Y^{1}\left(x^{1} X^{2} \cdot b_{2}\right)_{(-1)} x^{2} X_{1}^{3} \cdot b_{\underline{1}}^{\prime}\right] \\
\otimes\left[y_{1}^{3} Y^{2} \cdot\left(x^{1} X^{2} \cdot b_{\underline{2}}\right)_{(0)}\right]\left[y_{2}^{3} Y^{3} x^{3} X_{2}^{3} \cdot b_{\underline{2}}^{\prime}\right] .
\end{array}
$$

Finally, a bialgebra $B$ in ${ }_{H}^{H} \mathcal{Y} D$ is a Hopf algebra if there exists a morphism $\underline{S}$ : $B \rightarrow B$ in ${ }_{H}^{H} \mathcal{Y} D$ such that $\underline{S}\left(b_{1}\right) b_{2}=b_{1} S\left(b_{2}\right)=\underline{\varepsilon}_{B}(b) 1$ for all $b \in B$; we then say that $B$ is a braided Hopf algebra.

If $B \in{ }_{H}^{H} \mathcal{Y} D^{\mathrm{fd}}$ is a Hopf algebra then $B^{*}$ and ${ }^{*} B$ become Hopf algebras in ${ }_{H}^{H} \mathcal{Y} D$. We will compute the structure maps.

Proposition 5.1. Let $H$ be a quasi-Hopf algebra and $B$ a finite dimensional Hopf algebra in ${ }_{H}^{H} \mathcal{Y} D$. Then $B^{*}$ is a Hopf algebra in ${ }_{H}^{H} \mathcal{Y} D$ with the following structure maps:

$$
\begin{aligned}
\left(a^{*} \subseteq b^{*}\right)(b)= & \left\langle a^{*}, f^{2} \tilde{q}_{2}^{2} X^{3} S^{-1}\left(\tilde{q}^{1} X^{1}\left(p^{1} \cdot b_{2}\right)_{(-1)} p^{2}\right) \cdot b_{\underline{1}}\right\rangle \\
& \left\langle b^{*}, f^{1} \tilde{q}_{1}^{2} X^{2} \cdot\left(p^{1} \cdot b_{2}\right)_{(0)}\right\rangle \\
\Delta_{B^{*}}\left(b^{*}\right)= & \left\langle b^{*},\left[\left(g^{1} \cdot{ }_{j} b\right)_{(-1)} g^{2} \cdot{ }_{i} b\right]\left(g^{1} \cdot{ }_{j} b\right)_{(0)}\right\rangle^{i} b \otimes^{j} b \\
S_{B^{*}}\left(b^{*}\right)= & b^{*} \circ \underline{S}, u_{B^{*}}(1)=\underline{\varepsilon}_{B}, \quad \varepsilon_{B^{*}}\left(b^{*}\right)=b^{*}\left(1_{B}\right),
\end{aligned}
$$

for all $a^{*}, b^{*} \in B^{*}$ and $b \in B$, where $\left\{{ }_{i} b\right\}_{i=\overline{1, n}}$ is a basis in $B$ with dual basis $\left\{{ }^{i} b\right\}_{i=\overline{1, n}}$ in $B^{*}$. Similarly, ${ }^{*} B$ is a Hopf algebra in ${ }_{H}^{H} \mathcal{Y} D$ with the following structure maps:

$$
\begin{aligned}
\left({ }^{*} a \bar{\sigma}^{*} b\right)(b)= & \left\langle{ }^{*} a, S^{-1}\left(f^{2} q^{1}\left(x^{1} \tilde{p}^{1} S^{-1}\left(f^{1}\right) \cdot b_{\underline{2}}\right)_{(-1)} x^{2} \tilde{p}_{1}^{2}\right) \cdot b_{\underline{1}}\right\rangle \\
& \left\langle{ }^{*} b, S^{-1}\left(x^{3} \tilde{p}_{2}^{2}\right) q^{2} \cdot\left(x^{1} \tilde{p}^{1} S^{-1}\left(f^{1}\right) \cdot b_{\underline{2}}\right)_{(0)}\right\rangle \\
\Delta_{*_{B}}\left(b^{*}\right)= & \left\langle{ }^{*} b,\left[\left(S^{-1}\left(g^{2}\right) \cdot{ }_{j} b\right)_{(-1)} S^{-1}\left(g^{1}\right) \cdot{ }_{i} b\right]\left(S^{-1}\left(g^{2}\right) \cdot{ }_{j} b\right)_{(0)}\right\rangle^{i} b \otimes{ }^{j} b \\
S_{*_{B}}\left({ }^{*} b\right)= & { }^{*} b \circ \underline{S}, \quad u_{*_{B}}(1)=\underline{\varepsilon}_{B}, \quad \varepsilon^{*_{B}}\left({ }^{*} b\right)={ }^{*} b\left(1_{B}\right),
\end{aligned}
$$

for all ${ }^{*} a,{ }^{*} b \in{ }^{*} B$ and $b \in B$.

Proof. Follows easily by computing the morphisms in (5.15.3), using (4.184.21); the details are left to the reader.

Let $H$ be a Hopf algebra. It is well-known that $H$ becomes an algebra in the monoidal category ${ }_{H}^{H} \mathcal{Y} D$, with Yetter-Drinfeld structure given by

$$
h \triangleright h^{\prime}=h_{1} h^{\prime} S\left(h_{2}\right), \quad \lambda(h)=h_{1} \otimes h_{2}
$$

for all $h, h^{\prime} \in H$. Moreover, $H$ is quantum commutative as an algebra in ${ }_{H}^{H} \mathcal{Y} D$ (see for example [9]). Now, let $H$ be a quasi-Hopf algebra. In [7] a new multiplication $\circ$ on $H$ is introduced, given by the formula

$$
h \circ h^{\prime}=X^{1} h S\left(x^{1} X^{2}\right) \alpha x^{2} X_{1}^{3} h^{\prime} S\left(x^{3} X_{2}^{3}\right)
$$

Let $H_{0}$ be equal to $H$ as a vector space, with multiplication $\circ$. Then $H_{0}$ is a left $H$-module algebra with unit $\beta$ and left $H$-action given by

$$
h \triangleright h^{\prime}=h_{1} h^{\prime} S\left(h_{2}\right),
$$

for all $h, h^{\prime} \in H$. It was also shown in [6] that $H_{0}$ is an algebra in the category ${ }_{H}^{H} \mathcal{Y} D$, with $H$-coaction given by

$$
\lambda_{H}(h)=h_{(-1)} \otimes h_{(0)}=X^{1} Y_{1}^{1} h_{1} g^{1} S\left(q^{2} Y_{2}^{2}\right) Y^{3} \otimes X^{2} Y_{2}^{1} h_{2} g^{2} S\left(X^{3} q^{1} Y_{1}^{2}\right),
$$


where $f^{-1}=g^{1} \otimes g^{2}$ and $q=q_{R}=q^{1} \otimes q^{2}$ are the elements defined by (1.14) and (1.19). In addition, in [4] it was shown that $H_{0}$ is actually quantum commutative as an algebra in ${ }_{H}^{H} \mathcal{Y} D$.

If $(H, R)$ is quasitriangular, then $H_{0}$ is a Hopf algebra with bijective antipode in ${ }_{H} \mathcal{M}$, with the additional structure (see [6]):

$$
\begin{aligned}
& \underline{\Delta}(h)=h_{\underline{1}} \otimes h_{2} \\
& \quad=x^{1} X^{1} h_{1} g^{1} S\left(x^{2} R^{2} y^{3} X_{2}^{3}\right) \otimes x^{3} R^{1} \triangleright y^{1} X^{2} h_{2} g^{2} S\left(y^{2} X_{1}^{3}\right), \\
& \underline{\varepsilon}(h)=\varepsilon(h), \\
& \underline{S}(h)=X^{1} R^{2} p^{2} S\left(q^{1}\left(X^{2} R^{1} p^{1} \triangleright h\right) S\left(q^{2}\right) X^{3}\right),
\end{aligned}
$$

for all $h \in H$, where $R=R^{1} \otimes R^{2}$ and $f^{-1}=g^{1} \otimes g^{2}, p_{R}=p^{1} \otimes p^{2}$ and $q_{R}=q^{1} \otimes q^{2}$ are the elements defined by (1.14), (1.18) and (1.19). If we consider the left $H$ coaction

$$
\lambda_{H_{0}}(h)=R^{2} \otimes R^{1} \triangleright h,
$$

induced by (4.26), then $H_{0}$ becomes a Hopf algebra in ${ }_{H}^{H} \mathcal{Y} D$, with bijective antipode. From now on, we will refer to $H_{0}$ as a Hopf algebra in ${ }_{H} \mathcal{M}$, and, via the monoidal functor $\mathbb{F}:{ }_{H} \mathcal{M} \rightarrow{ }_{H}^{H} \mathcal{Y} D$, in ${ }_{H}^{H} \mathcal{Y} D$, with structure maps (5.18), (5.19) and (5.21 5.24).

If $H$ is finite dimensional, then $H_{0}^{*}$ is also a Hopf algebra in ${ }_{H}^{H} \mathcal{Y} D$. By Theorem 3.2 and (1.37), $H_{0}^{*}$ is a Yetter-Drinfeld module via

$$
\begin{aligned}
& (h \mapsto \varphi)\left(h^{\prime}\right)=\varphi\left(S(h) \triangleright h^{\prime}\right) \\
& \lambda_{H_{0}^{*}}(\varphi)=R^{2} \otimes R^{1} \mapsto \varphi
\end{aligned}
$$

for all $\varphi \in H^{*}$ and $h, h^{\prime} \in H$. The structure of $H_{0}^{*}$ as a Hopf algebra in ${ }_{H}^{H} \mathcal{Y} D$ is given by:

$$
\begin{aligned}
(\varphi \underline{\circ} \Psi)(h) & =\left\langle\varphi, f^{2} \bar{R}^{2} \triangleright h_{\underline{1}}\right\rangle\left\langle\Psi, f^{1} \bar{R}^{1} \triangleright h_{\underline{2}}\right\rangle, \\
1_{H_{0}^{*}} & =\underline{\varepsilon}, \\
\Delta_{H_{0}^{*}}(\varphi) & =\left\langle\varphi,\left(R^{2} g^{2} \triangleright_{i} e\right) \circ\left(R^{1} g^{1} \triangleright_{j} e\right)\right\rangle^{i} e \otimes^{j} e, \\
\varepsilon_{H_{0}^{*}}(\varphi) & =\varphi(\beta), \\
S_{H_{0}^{*}}(\varphi) & =\varphi \circ \underline{S},
\end{aligned}
$$

for all $h \in H$ and $\varphi, \Psi \in H^{*}$, where $R^{-1}=\sum \bar{R}^{1} \otimes \bar{R}^{2},\left\{{ }_{i} e\right\}_{i=\overline{1, n}}$ is a basis of $H$ and $\left\{{ }^{i} e\right\}_{i=\overline{1, n}}$ the corresponding dual basis of $H^{*}$. The left dual ${ }^{*} H_{0}$ of $H_{0}$ is also a Hopf algebra in ${ }_{H}^{H} \mathcal{Y} D$. First, by Theorem 3.2 and (1.37), ${ }^{*} H_{0}$ is a Yetter-Drinfeld module via

$$
\begin{aligned}
& (h \succ \varphi)\left(h^{\prime}\right)=\varphi\left(S^{-1}(h) \triangleright h^{\prime}\right) \\
& \lambda_{* H_{0}}(\varphi)=R^{2} \otimes R^{1} \succ \varphi
\end{aligned}
$$

for all $\varphi \in H^{*}$ and $h, h^{\prime} \in H$. Then the structure of ${ }^{*} H_{0}$ as a Hopf algebra in ${ }_{H}^{H} \mathcal{Y} D$ is given by the formulae

$$
\begin{aligned}
& (\varphi \bar{\circ} \Psi)(h)=\left\langle\varphi, S^{-1}\left(f^{2} \bar{R}^{2}\right) \triangleright h_{\underline{1}}\right\rangle\left\langle\Psi, S^{-1}\left(f^{1} \bar{R}^{1}\right) \triangleright h_{\underline{2}}\right\rangle, \\
& 1_{* H_{0}}=\underline{\varepsilon},
\end{aligned}
$$




$$
\begin{aligned}
& \Delta^{*} H_{0}(\varphi)=\left\langle\varphi,\left[S^{-1}\left(R^{2} g^{2}\right) \triangleright_{i} e\right] \circ\left[S^{-1}\left(R^{1} g^{1}\right){ }_{j} e\right]\right\rangle^{i} e \otimes^{j} e, \\
& \varepsilon^{*} H_{0}(\varphi)=\varphi(\beta), \\
& S_{*} H_{0}(\varphi)=\varphi \circ \underline{S},
\end{aligned}
$$

for all $\varphi \in H^{*}$ and $h, h^{\prime} \in H$. From (4.5) we know that $\Theta_{H_{0}}: H_{0}^{*} \rightarrow{ }^{*} H_{0}$ is an isomorphism of Yetter-Drinfeld modules. In this particular case, $\Theta_{H_{0}}$ is given by

$$
\begin{array}{cc}
\Theta_{H_{0}}(\varphi) \frac{4.84 .26}{=} & \left\langle\varphi, S\left(p^{1}\right) f^{2} R^{1} g^{1} \triangleright_{j} e\right\rangle \\
\left\langle{ }^{j} e, S\left(q^{2}\right) S^{-1}\left(q^{1} S^{-1}\left(f^{1} R^{2} g^{2}\right) p^{2}\right) \triangleright_{i} e\right\rangle^{i} e \\
\frac{1.37}{=} & \left\langle\varphi, S\left(R^{1} p^{1}\right) \triangleright{ }_{j} e\right\rangle\left\langle{ }^{j} e, S^{-1}\left(q^{1} R^{2} p^{2} S^{2}\left(q^{2}\right)\right) \triangleright_{i} e\right\rangle^{i} e \\
\frac{1.35}{=} & \left\langle\varphi, S^{-1}\left(u^{-1}\right) \triangleright{ }_{i} e\right\rangle^{i} e=u^{-1} \succ \varphi
\end{array}
$$

for all $\varphi \in H_{0}^{*}$. Since $S^{2}(u)=u$ it is easy to see that $\Theta_{H_{0}}^{-1}(\varphi)=u \mapsto \varphi$ for all $\varphi \in{ }^{*} H_{0}$.

Proposition 5.2. Let $(H, R)$ be a triangular quasi-Hopf algebra. With notation as above, $\Theta_{H_{0}}: H_{0}^{*} \rightarrow{ }^{*} H_{0}$ is a braided Hopf algebra isomorphism.

Proof. It is well known that in a symmetric monoidal category $\mathcal{C}$ the canonical isomorphism $\Theta_{B}$, where $B$ is a Hopf algebra in $\mathcal{C}$, provides a braided Hopf algebra isomorphism between $B^{*}$ and ${ }^{*} B$. Since $(H, R)$ is triangular, the category ${ }_{H} \mathcal{M}^{\mathrm{fd}}$ is symmetric, and this finishes the proof.

Remark 5.3. If $(H, R)$ is not triangular then $\Theta_{H_{0}}$ is, in general, not an algebra or a coalgebra morphism. Indeed, if $(H, R)$ is an arbitrary quasitriangular quasi-Hopf algebra then computations similar to the ones presented above show that

$$
\begin{aligned}
& \Theta_{H_{0}}\left(\left(\bar{r}^{1} \bar{R}^{2} \mapsto \varphi\right) \varrho\left(\bar{r}^{2} \bar{R}^{1} \mapsto \Psi\right)\right)=\Theta_{H_{0}}(\varphi) \bar{\circ} \Theta_{H_{0}}(\Psi), \\
& \left(\Delta_{*_{H_{0}}} \Theta_{H_{0}}\right)(\varphi)=R_{21} R \succ\left(\Theta_{H_{0}} \otimes \Theta_{H_{0}}\right)\left(\Delta_{H_{0}^{*}}(\varphi)\right)
\end{aligned}
$$

for any $\varphi, \Psi \in H_{0}^{*}$, where $\bar{r}^{1} \otimes \bar{r}^{2}$ is another copy of $R^{-1}$ and we extend the action of $H$ on ${ }^{*} H_{0}$ to an action of $H \otimes H$ on ${ }^{*} H_{0} \otimes{ }^{*} H_{0}$.

Let $H$ be a quasi-Hopf algebra. Then $H^{*}$ is an $(H, H)$-bimodule, by

$$
\left\langle h \rightarrow \varphi, h^{\prime}\right\rangle=\varphi\left(h^{\prime} h\right), \quad\left\langle\varphi<h, h^{\prime}\right\rangle=\varphi\left(h h^{\prime}\right) .
$$

The convolution $\langle\varphi \Psi, h\rangle=\varphi\left(h_{1}\right) \psi\left(h_{2}\right), h \in H$, is a multiplication on $H^{*}$; it is not associative, but only quasi-associative:

$$
(\varphi \psi) \xi=\left(X^{1}-\varphi<x^{1}\right)\left[\left(X^{2} \rightarrow \psi<x^{2}\right)\left(X^{3}-\xi<x^{3}\right)\right], \quad \forall \varphi, \psi, \xi \in H^{*} .
$$

In addition, for all $h \in H$ and $\varphi, \psi \in H^{*}$ we have that

$$
h \rightarrow(\varphi \psi)=\left(h_{1} \rightarrow \varphi\right)\left(h_{2} \rightarrow \psi\right) \text { and }(\varphi \psi)<h=\left(\varphi<h_{1}\right)\left(\psi<h_{2}\right) .
$$

By [3] if $H$ is a finite dimensional quasitriangular quasi-Hopf algebra then on the dual of $H$ there exists another structure of Hopf algebra in ${ }_{H}^{H} \mathcal{Y} D$, denoted by $\underline{H}^{*}$. The structure of $\underline{H}^{*}$ as a Yetter-Drinfeld module is given by the formulae

$$
\begin{aligned}
h \cdot \varphi & =h_{1} \rightarrow \varphi<S^{-1}\left(h_{2}\right), \\
\lambda_{\underline{H}^{*}}(\varphi) & =R^{2} \otimes R^{1} \cdot \varphi,
\end{aligned}
$$


for all $h \in H, \varphi \in H^{*}$. The structure of $\underline{H}^{*}$ as a Hopf algebra in ${ }_{H}^{H} \mathcal{Y} D$ is given by:

$$
\begin{gathered}
\varphi \bullet \Psi=\left(x^{1} X^{1}-\varphi<S^{-1}\left(f^{2} x_{2}^{3} Y^{3} R^{1} X^{2}\right)\right) \\
\left(x^{2} Y^{1} R_{1}^{2} X_{1}^{3} \rightarrow \Psi \leftarrow S^{-1}\left(f^{1} x_{1}^{3} Y^{2} R_{2}^{2} X_{2}^{3}\right)\right), \\
\Delta_{\underline{H}^{*}}(\varphi)=X_{1}^{1} p^{1} \rightarrow \varphi_{2} \leftarrow S^{-1}\left(X_{2}^{1} p^{2}\right) \otimes X^{2}-\varphi_{1} \leftarrow S^{-1}\left(X^{3}\right), \\
\varepsilon_{\underline{H}^{*}}(\varphi)=\varphi\left(S^{-1}(\alpha)\right), \\
S_{\underline{H}^{*}}(\varphi)=Q^{1} q^{1} R^{2} x^{2} \cdot\left[p^{1} P^{2} S\left(Q^{2}\right)\right. \\
\left.\quad-\bar{S}^{-1}(\varphi) \leftarrow S\left(q^{2} R^{1} x^{1} P^{1}\right) x^{3} S^{-1}\left(p^{2}\right)\right],
\end{gathered}
$$

for all $\varphi, \Psi \in H^{*}$, where $Q^{1} \otimes Q^{2}$ is another copy of $q_{R}$ and $\bar{S}^{-1}(\varphi)=\varphi \circ S^{-1}$ for any $\varphi \in H^{*}$. The unit element is $\varepsilon$.

Proposition 5.4. Let $(H, R)$ be a finite dimensional triangular quasi-Hopf algebra. Then ${ }^{*} H_{0}$ and $\underline{H}^{* c o p}$ are isomorphic as braided Hopf algebras.

Proof. Since $(H, R)$ is triangular it follows that $c_{N, M}^{-1}=c_{M, N}$, so $\underline{H}^{* c o p}$ is a Hopf algebra in ${ }_{H}^{H} \mathcal{Y} D$. On the other hand, both ${ }^{*} H_{0}$ and $\underline{H}^{* c o p}$ are Hopf algebras in ${ }_{H}^{H} \mathcal{Y} D$ as images, through the functor $\mathbb{F}$ defined by (4.26), of corresponding objects in ${ }_{H} \mathcal{M}$. Therefore, it suffices to prove that ${ }^{*} H_{0}$ and $\underline{H}^{* c o p}$ are isomorphic as Hopf algebras in ${ }_{H} \mathcal{M}$. To this end we claim that $\mu:{ }^{*} H_{0} \rightarrow \underline{H}^{* c o p}$ given by

$$
\mu(\varphi)=g^{1} \rightarrow \varphi<S^{-1}\left(g^{2}\right)
$$

is a Hopf algebra isomorphism in ${ }_{H} \mathcal{M}$. In fact, $\mu$ is $H$-linear since

$$
\begin{array}{ccl}
\mu(h \succ \varphi)\left(h^{\prime}\right) & = & \left\langle\varphi, S^{-1}(h) \triangleright\left(S^{-1}\left(g^{2}\right) h^{\prime} g^{1}\right)\right\rangle \\
& 1.11 & \left\langle\varphi, S^{-1}\left(h_{2} g^{2}\right) h^{\prime} h_{1} g^{1}\right\rangle \\
& =.41 & \left\langle\mu(\varphi), S^{-1}\left(h_{2}\right) h^{\prime} h_{1}\right\rangle=(h \cdot \mu(\varphi))\left(h^{\prime}\right)
\end{array}
$$

for all $\varphi \in H^{*}$ and $h, h^{\prime} \in H$. It is also an algebra morphism in ${ }_{H} \mathcal{M}$ since

$$
\begin{aligned}
& \mu(\varphi \bar{\circ} \Psi)(h) \\
& \text { 5.345.211.11 }\left\langle\varphi, S^{-1}\left(f^{2} \bar{R}^{2}\right) \triangleright x^{1} X^{1} S^{-1}\left(F^{2} g_{2}^{2} \mathbf{G}^{2}\right) h_{1} g_{1}^{1} G^{1}\right. \\
& \left.S\left(x^{2} R^{2} y^{3} X_{2}^{3}\right)\right\rangle\left\langle\Psi, S^{-1}\left(f^{1} \bar{R}^{1}\right) x^{3} R^{1} \triangleright y^{1} X^{2}\right. \\
& \left.S^{-1}\left(F^{1} g_{1}^{2} \mathbf{G}^{1}\right) h_{2} g_{2}^{1} G^{2} S\left(y^{2} X_{1}^{3}\right)\right\rangle \\
& \frac{1.111 .91 .17}{=}\left\langle\varphi, S^{-1}\left(f^{2} \bar{R}^{2}\right) \triangleright x^{1} S^{-1}\left(F^{2} X^{3} g^{2}\right) h_{1} X_{1}^{1} g_{(1,1)}^{1} \mathbf{G}_{1}^{1} G^{1}\right. \\
& \left.S\left(x^{2} R^{2} y^{3}\right)\right\rangle\left\langle\Psi, S^{-1}\left(f^{1} \bar{R}^{1}\right) x^{3} R^{1} \triangleright y^{1}\right. \\
& \left.S^{-1}\left(F^{1} X^{2} g_{2}^{1} \mathbf{G}^{2}\right) h_{2} X_{2}^{1} g_{(1,2)}^{1} \mathbf{G}_{2}^{1} G^{2} S\left(y^{2}\right)\right\rangle \\
& \left\langle\varphi, S^{-1}\left(f^{2} \bar{R}^{2}\right) \triangleright x^{1} S^{-1}\left(F^{2} X^{3} g^{2}\right) h_{1} X_{1}^{1} y^{1} g_{1}^{1}\right. \\
& \left.\mathbf{G}^{1} S\left(x^{2} R^{2}\right)\right\rangle\left\langle\Psi, S^{-1}\left(f^{1} \bar{R}^{1}\right) \triangleright S^{-1}\left(F^{1} X^{2} y^{3} g_{(2,2)}^{1}\right.\right. \\
& \left.\left.\mathbf{G}_{2}^{2} G^{2} S\left(x_{1}^{3} R_{1}^{1}\right)\right) h_{2} X_{2}^{1} y^{2} g_{(2,1)}^{1} \mathbf{G}_{1}^{2} G^{1} S\left(x_{2}^{3} R_{2}^{1}\right)\right\rangle \\
& \stackrel{1.111 .371 .31}{=}\left\langle\varphi, S^{-1}\left(f^{2} \bar{R}^{2}\right) \triangleright x^{1} S^{-1}\left(F^{2} X^{3} g^{2}\right) h_{1} X_{1}^{1} y^{1} R^{2} g_{2}^{1} \mathbf{G}^{2} S\left(x^{2}\right)\right\rangle \\
& \left\langle\Psi, S^{-1}\left(f^{1} \bar{R}^{1}\right) \triangleright S^{-1}\left(F^{1} X^{2} y^{3} R_{2}^{1}\left(g_{1}^{1} \mathbf{G}^{1} S\left(x^{3}\right)\right)_{2} G^{2}\right)\right. \\
& \left.h_{2} X_{2}^{1} y^{2} R_{1}^{1}\left(g_{1}^{1} \mathbf{G}^{1} S\left(x^{3}\right)\right){ }_{1} G^{1}\right\rangle
\end{aligned}
$$




$$
\begin{aligned}
& \frac{1.91 .17}{=}\left\langle\varphi, S^{-1}\left(f^{2} \bar{R}^{2}\right) \triangleright S^{-1}\left(F^{2} X^{3} x^{3} g_{2}^{2} \mathbf{G}^{2}\right) h_{1} X_{1}^{1} y^{1} R^{2}\right. \\
& \left.x^{2} g_{1}^{2} \mathbf{G}^{1}\right\rangle\left\langle\Psi, S^{-1}\left(f^{1} \bar{R}^{1}\right) \triangleright S^{-1}\left(F^{1} X^{2} y^{3} R_{2}^{1} x_{2}^{1} g_{2}^{1} G^{2}\right) h_{2} X_{2}^{1} y^{2} R_{1}^{1} x_{1}^{1} g_{1}^{1} G^{1}\right\rangle \\
& 1.11 \text { twice } \quad\left\langle\varphi, S^{-1}\left(F^{2} X^{3} x^{3} \bar{R}_{2}^{2} g^{2}\right) h_{1} X_{1}^{1} y^{1} R^{2} x^{2} \bar{R}_{1}^{2} g^{1}\right\rangle \\
& = \\
& \left\langle\Psi, S^{-1}\left(R^{1} x^{1} \bar{R}^{1}\right) \triangleright S^{-1}\left(F^{1} X^{2} y^{3} G^{2}\right) h_{2} X_{2}^{1} y^{2} G^{1}\right\rangle \\
& \frac{1.301 .3}{=}\left\langle\varphi, S^{-1}\left(F^{2} x_{2}^{3} X^{3} \bar{R}^{2} Y^{2} g^{2}\right) h_{1} x^{1} Y^{1} g^{1}\right\rangle \\
& \left\langle\Psi, S^{-1}\left(\bar{R}^{1} Y^{3}\right) \triangleright S^{-1}\left(F^{1} x_{1}^{3} X^{2} G^{2}\right) h_{2} x^{2} X^{1} G^{1}\right\rangle \\
& \left.1.11 \mathrm{R}^{-1}=\mathrm{R}_{21}\right) \quad\left\langle\mu(\varphi), S^{-1}\left(F^{2} x_{2}^{3} X^{3} R^{1} Y^{2}\right) h_{1} x^{1} Y^{1}\right\rangle \\
& = \\
& \left\langle\mu(\Psi), S^{-1}\left(F^{1} x_{1}^{3} X^{2} R_{2}^{2} Y_{2}^{3}\right) h_{2} x^{2} X^{1} R_{1}^{2} Y_{1}^{3}\right\rangle \\
& \frac{5.43}{=} \quad(\mu(\varphi) \bullet \mu(\Psi))(h)
\end{aligned}
$$

for all $\varphi, \Psi \in H^{*}, h \in H$, and $\mu\left(1^{*} H_{0}\right)=\mu(\varepsilon)=\varepsilon=1_{H^{* c o p}}$. It remains to show that $\mu$ is a coalgebra morphism and a bijection. To this end, we calculate for any $\varphi \in H^{*}$ :

$$
\begin{aligned}
& (\mu \otimes \mu) \Delta_{*} H_{0}=\left\langle\varphi,\left(S^{-1}\left(R^{2} g^{2}\right) \triangleright_{i} e\right) \circ\left(S^{-1}\left(R^{1} g^{1}\right) \triangleright_{j} e\right)\right\rangle \\
& G^{1} \rightarrow{ }^{i} e<S^{-1}\left(G^{2}\right) \otimes \mathbf{G}^{1} \rightarrow{ }^{j} e<S^{-1}\left(\mathbf{G}^{2}\right) \\
& \stackrel{5.181 .37}{=}\left\langle\varphi, X^{1}\left[R^{2} S^{-1}\left(g^{1}\right) \triangleright S^{-1}\left(G^{2}\right)_{i} e G^{1}\right] S\left(x^{1} X^{2}\right) \alpha x^{2} X_{1}^{3}\right. \\
& \left.\left[R^{1} S^{-1}\left(g^{2}\right) \triangleright S^{-1}\left(\mathbf{G}^{2}\right){ }_{j} e \mathbf{G}^{1}\right] S\left(x^{3} X_{2}^{3}\right)\right\rangle^{i} e \otimes{ }^{j} e \\
& \stackrel{1.11}{=}\left\langle\varphi, X^{1} R_{1}^{2} S^{-1}\left(g_{2}^{1} G^{2}\right)_{i} e g_{1}^{1} G^{1} S\left(x^{1} X^{2} R_{2}^{2}\right) \alpha x^{2}\right. \\
& \left.\left[X^{3} R^{1} S^{-1}\left(g^{2}\right) \triangleright S^{-1}\left(\mathbf{G}^{2}\right)_{j} e \mathbf{G}^{1}\right] S\left(x^{3}\right)\right\rangle^{i} e \otimes^{j} e \\
& \left\langle\varphi, X^{1} S^{-1}\left(y^{2} r^{2} g_{2}^{2} G^{2}\right)_{i} e y^{1} g^{1} S\left(x^{1} R^{2} X^{3}\right) \alpha x^{2}\right. \\
& \left.\left[R^{1} X^{2} S^{-1}\left(y^{3} r^{1} g_{1}^{2} G^{1}\right) \triangleright S^{-1}\left(\mathbf{G}^{2}\right)_{j} e \mathbf{G}^{1}\right] S\left(x^{3}\right)\right\rangle^{i} e \otimes^{j} e \\
& \text { 1.91.17 }\left\langle\varphi, S^{-1}\left(y^{2} r^{2} X^{3} g^{2}\right)_{i} e y^{1} X^{1} g_{1}^{1} G^{1} S\left(x^{1} R^{2}\right) \alpha x^{2}\right. \\
& \left.\left[R^{1} S^{-1}\left(y^{3} r^{1} X^{2} g_{2}^{1} G^{2}\right) \triangleright S^{-1}\left(\mathbf{G}^{2}\right)_{j} e \mathbf{G}^{1}\right] S\left(x^{3}\right)\right\rangle^{i} e \otimes{ }^{j} e \\
& \text { 1.371.311.11 }\left\langle\varphi, S^{-1}\left(R_{2}^{2} X^{3} g^{2}\right)_{i} e R_{1}^{2} X^{2} g_{2}^{1} G^{2} S\left(x^{1}\right) \alpha x^{2}\right. \\
& \left.S^{-1}\left(R_{2}^{1} X_{2}^{1} g_{(1,2)}^{1} G_{2}^{1} \mathbf{G}^{2}\right)_{j} e R_{1}^{1} X_{1}^{1} g_{(1,1)}^{1} G_{1}^{1} \mathbf{G}^{1} S\left(x^{3}\right)\right\rangle^{i} e \otimes{ }^{j} e \\
& \text { 1.91.171.1 }\left\langle\varphi, S^{-1}\left(R_{2}^{2} X^{3} g^{2}\right)_{i} e R_{1}^{2} X^{2} x^{3} g_{(2,2)}^{1} G_{2}^{2} \mathbf{G}^{2} \alpha\right. \\
& \left.S^{-1}\left(R_{2}^{1} X_{2}^{1} x^{2} g_{(2,1)}^{1} G_{1}^{2} \mathbf{G}^{1}\right)_{j} e R_{1}^{1} X_{1}^{1} x^{1} g_{1}^{1} G^{1} S\left(x^{3}\right)\right\rangle^{i} e \otimes{ }^{j} e \\
& \frac{1.161 .5}{=}\left\langle\varphi, S^{-1}\left(R_{2}^{2} X^{3} g^{2}\right)_{i} e R_{1}^{2} X^{2} x^{3} S^{-1}\left(R_{2}^{1} X_{2}^{1} x^{2} \beta\right)\right. \\
& \left\langle\mu(\varphi), S^{-1}\left(X^{3}\right)_{i} e X^{2} S^{-1}\left(X_{2}^{1} p^{2}\right)_{j} e X_{1}^{1} p^{1}\right\rangle \\
& R^{2} \cdot{ }^{i} e \otimes R^{1} \cdot{ }^{j} e \\
& \stackrel{5.44}{\left.\mathrm{R}^{-1}=\mathrm{R}_{21}\right)} \quad c_{\underline{H}^{*}, \underline{H}^{*}}^{-1}\left(\Delta_{\underline{H}^{*}}(\mu(\varphi))\right)=\Delta_{\underline{H}^{* c o p}}(\mu(\varphi))
\end{aligned}
$$

as needed. Obviously $\varepsilon \mu=\varepsilon$. It is easy to see that $\mu$ is bijective with inverse

$$
\mu^{-1}(\varphi)=f^{1} \rightarrow \varphi<S^{-1}\left(f^{2}\right)
$$

for any $\varphi \in H^{*}$. Thus, the proof is complete. 
Corollary 5.5. If $(H, R)$ is a finite dimensional triangular quasi-Hopf algebra then $H_{0}^{*} \cong{ }^{*} H_{0} \cong \underline{H}^{* \text { cop }}$ as braided Hopf algebras.

\section{ACKNOWLEDGEMENT}

The authors wish to thank the referee for his suggestion to use the centre construction to simplify the proofs of the results in Sections 2 and 3

\section{REFERENCES}

[1] D. Altschuler and A. Coste, Quasi-quantum groups, knots, three-manifolds, and topological field theory, Comm. Math. Phys. 150 (1992), 83-107.

[2] N. Andruskiewitsh and M. Graña, Braided Hopf algebras over abelian finite groups, Bol. Acad. Ciencias (Còrdoba) 63 (1999), 45-78.

[3] D. Bulacu and S. Caenepeel, The quantum double for quasitriangular quasi-Hopf algebras, Comm. Algebra 31 (2003), 1403-1425.

[4] D. Bulacu, S. Caenepeel and F. Panaite, More properties of Yetter-Drinfeld modules over quasi-Hopf algebras, in "Hopf algebras in non-commutative geometry and physics", Caenepeel S. and Van Oystaeyen, F. (eds.), Lecture Notes Pure Appl. Math. 239, Dekker, New York, to appear in October 2004.

[5] D. Bulacu and E. Nauwelaerts, Quasitriangular and ribbon quasi-Hopf algebras, Comm. Algebra 31 (2003), 1-16.

[6] D. Bulacu and E. Nauwelaerts, Radford's biproduct for quasi-Hopf algebras and bosonization, J. Pure Appl. Algebra 174 (2002), 1-42.

[7] D. Bulacu, F. Panaite and F. Van Oystaeyen, Quasi-Hopf algebra actions and smash products, Comm. Algebra 28 (2000), 631-651.

[8] D. Bulacu, F. Panaite and F. Van Oystaeyen, Quantum traces and quantum dimensions for quasi-Hopf algebras, Comm. Algebra 27 (1999), 6103-6122.

[9] S. Caenepeel, F. Van Oystaeyen and Y. H. Zhang, Quantum Yang-Baxter module algebras, K-Theory 8 (1994), 231-255.

[10] R. Dijkgraaf, V. Pasquier and P. Roche, Quasi-Hopf algebras, group cohomology and orbifold models, Nuclear Phys. B Proc. Suppl. 18 B (1990), 60-72.

[11] V. G. Drinfeld, Quasi-Hopf algebras, Leningrad Math. J. 1 (1990), 1419-1457.

[12] F. Hausser and F. Nill, Diagonal crossed products by duals of quasi-quantum groups, Rev. Math. Phys. 11 (1999), 553-629.

[13] F. Hausser and F. Nill, Doubles of quasi-quantum groups, Comm. Math. Phys. 199 (1999), $547-589$.

[14] C. Kassel, "Quantum Groups", Graduate Texts in Mathematics 155, Springer Verlag, Berlin, 1995.

[15] S. Majid, Quantum double for quasi-Hopf algebras, Lett. Math. Phys. 45 (1998), 1-9.

[16] S. Majid, "Foundations of quantum group theory", Cambridge Univ. Press, 1995.

[17] D. E. Radford and J. Towber, Yetter-Drinfeld categories associated to an arbitrary bialgebra, J. Pure Appl. Algebra 87 (1993), 259-279.

[18] P. Schauenburg, Hopf modules and the double of a quasi-Hopf algebra, Trans. Amer. Math. Soc. 354 (2002), 3349-3378.

[19] M. E. Sweedler, "Hopf algebras", Benjamin, New York, 1969.

[20] M. Takeuchi, Finite Hopf algebras in braided tensor categories, J. Pure Appl. Algebra 138 (1999), 59-82.

Faculty of Mathematics, University of Bucharest, RO-010014 Bucharest 1, Romania

E-mail address: dbulacu@al.math.unibuc.ro

Faculty of Applied Sciences, Vrije Universiteit Brussel, VUB, B-1050 Brussels, Belgium

E-mail address: scaenepe@vub.ac.be

URL: http://homepages.vub.ac.be/ scaenepe/

Institute of Mathematics of the Romanian Academy, PO-Box 1-764, RO-70700 BuchaREST, ROMANIA

E-mail address: Florin.Panaite@imar.ro 